Mon. Not. R. Astron. Soc. 000, 田 14 (2000) Printed 25 October $2018 \quad$ (MN LATEX style file v1.4)

\title{
Dwarf Galaxy Rotation Curves and the Core Problem of Dark Matter Haloes
}

\author{
Frank C. van den Bosch ${ }^{1,2 \star}$ and Rob A. Swaters ${ }^{3}$ \\ ${ }^{1}$ Department of Astronomy, University of Washington, Seattle, WA 98195, USA \\ ${ }^{2}$ Max-Planck Institut für Astrophysik, Karl Schwarzschild Str. 1, Postfach 1317, 85741 Garching, Germany \\ ${ }^{3}$ Carnegie Institution of Washington, Washington DC 20015, USA
}

\begin{abstract}
The standard cold dark matter (CDM) model has recently been challenged by the claim that dwarf galaxies have dark matter haloes with constant density cores, whereas CDM predicts haloes with steeply cusped density distributions. Consequently, numerous alternative dark matter candidates have recently been proposed. In this paper, we scrutinize the observational evidence for the incongruity between dwarf galaxies and the CDM model. To this end, we analyze the rotation curves of 20 late-type dwarf galaxies studied by Swaters (1999). Taking the effects of beam-smearing and adiabatic contraction into account, we fit mass models to these rotation curves with dark matter haloes with different cusp slopes, ranging from constant density cores to $r^{-2}$ cusps. Even though the effects of beam smearing are small for these data, the uncertainties in the stellar mass-to-light ratio and the limited spatial sampling of the halo's density distribution hamper a unique mass decomposition. Consequently, the rotation curves in our sample cannot be used to discriminate between dark haloes with constant density cores and $r^{-1}$ cusps. We show that the dwarf galaxies analyzed here are consistent with cold dark matter haloes in a $\Lambda \mathrm{CDM}$ cosmology, and that there is thus no need to abandon the idea that dark matter is cold and collisionless. However, the data is also consistent with any alternative dark matter model that produces dark matter haloes with central cusps less steep than $r^{-1.5}$. In fact, we argue that based on existing HI rotation curves alone at best weak limits can be obtained on cosmological parameters and/or the nature of the dark matter. In order to make progress, rotation curves with higher spatial resolution and independent measurements of the mass-to-light ratio of the disk are required.
\end{abstract}

Key words: dark matter - galaxies: haloes - galaxies: kinematics and dynamics galaxies: fundamental parameters - galaxies: structure.

\section{INTRODUCTION}

The standard cosmological model for structure formation combines an inflationary Universe with hierarchical growth of structures that originate from small fluctuations in the cosmic mass distribution. In addition to baryonic matter, this model requires non-baryonic dark matter and possibly some form of vacuum energy or quintessence. Unfortunately, the nature of the dark matter, which is the dominant mass component, still remains unknown. A large number of candidates have been proposed of which cold dark matter (CDM) has been the most popular. Because CDM particles have a negligible thermal velocity with respect to the Hubble flow, the original phase-space density of cold dark matter

* Hubble Fellow is extremely high. Numerical simulations have shown that a small fraction of this material remembers its initial phasespace density even after it collapses to form a bound object. This low-entropy material settles in the centers of virialized dark haloes, thus creating steeply cusped density profiles, and causing a large fraction of haloes to survive as substructure inside larger haloes (e.g., Navarro, Frenk \& White 1996; Fukushige \& Makino 1997; Moore et al. 1998, 1999b; Ghigna et al. 1998; Klypin et al. 1999a; White \& Springel 1999).

These characteristics of CDM haloes, however, seem to disagree with a number of observations. First, the number of sub-haloes around a typical Milky Way galaxy, as identified by satellite galaxies, is an order of magnitude smaller than predicted by CDM (Kauffmann, White \& Guiderdoni 1993; Klypin et al. 1999b; Moore et al. 1999a). Secondly, the observed rotation curves of dwarf and low surface bright-

(C) 2000 RAS 
van den Bosch \&6 Swaters

ness (LSB) galaxies seem to indicate that their dark matter haloes have constant density cores instead of steep cusps (Flores \& Primack 1994; Moore 1994; Burkert 1995; Burkert \& Silk 1997; McGaugh \& de Blok 1998; Stil 1999; Moore et al. 1999b; Dalcanton \& Bernstein 2000; Firmani et al. 2001). In view of these discrepancies, numerous alternatives to the CDM paradigm have recently been proposed. These include broken scale-invariance (hereafter BSI; Kamionkowski \& Liddle 2000; White \& Croft 2000), warm dark matter (hereafter WDM; Sommer-Larsen \& Dolgov 1999; Hogan \& Dalcanton 2000), scalar field dark matter (hereafter SFDM; Peebles \& Vilenkin 1999; Hu \& Peebles 1999; Peebles 2000; Matos, Siddharta \& Urena-López 2000), and various sorts of self-interacting or annihilating dark matter (hereafter SIDM; Carlson, Machacek \& Hall 1992; Spergel \& Steinhardt 2000; Mohapatra \& Teplitz 2000; Firmani et al. 2000; Goodman 2000; Kaplinghat, Knox \& Turner 2000; Bento et al. 2000). Whereas particle physics does not prefer CDM over for instance WDM, SFDM, or SIDM, the former has the advantage over the latter that it has no free parameters. Furthermore, most of these alternatives seem unable to solve both problems simultaneously (Moore et al. 1999b; Hogan \& Dalcanton 2000; Colín, Avila-Reese \& Valenzuela 2000; Dalcanton \& Hogan 2000), and often the alternatives face their own problems (Spergel \& Steinhardt 2000; Hannestad 1999; Burkert 2000; Moore et al. 2000; Yoshida et al. 2000; Kochanek \& White 2000; Miralde-Escude 2000; Sellwood 2000).

As an alternative to modifying the nature of the dark matter, the sub-structure and core problems might be solved once additional baryonic physics are taken into account. Several studies have suggested that processes such as reionization and supernova feedback can help to suppress star formation and to decrease central densities in low-mass dark matter haloes (e.g., Navarro, Eke \& Frenk 1996; Gelato \& Sommer-Larsen 1999; van den Bosch et al. 2000; Bullock, Kravtsov \& Weinberg 2000; Binney, Gerhard \& Silk 2001). Whereas these processes may indeed help to solve the problem with the over-abundance of satellite galaxies, the suggestion that feedback processes can actually destroy steep central cusps seems somewhat contrived in light of more detailed simulations. For instance, as shown by Navarro, Eke $\&$ Frenk, the effects are only substantial if large fractions of baryonic mass are expelled, which seems hard to reconcile with the low ejection efficiencies found in more detailed hydro-dynamical simulations (e.g., Mac-Low \& Ferrara 1999; Strickland \& Stevens 2000).

It is evident from the above discussion that the longtime popular CDM paradigm is currently facing its biggest challenge to date. However, before abandoning CDM on the grounds that it is inconsistent with observations, it is worthwhile to more closely examine the observational evidence against it. In this paper we scrutinize CDM's most persistent problem: the claim that the dark haloes of dwarf galaxies, as inferred from their rotation curves, are inconsistent with CDM predictions. The main motivation for this work is that recent work on the rotation curves of LSB galaxies has shown that once data with sufficient resolution is obtained, or the effects of beam smearing are properly taken into account, the inner rotation curves are significantly steeper and allow for more centrally concentrated dark matter haloes (Swaters 1999; van den Bosch et al. 2000; Swaters, Madore
\& Trewhella 2000). In fact, these studies have pointed out that, in contrast with previous claims, current data on LSB rotation curves are consistent with CDM predictions.

Here we analyze a set of HI rotation curves of a sample of 20 late-type dwarf galaxies. Taking beam smearing and adiabatic contraction of the dark matter into account, we investigate whether the rotation curves of the galaxies in our sample are consistent with CDM haloes. Although we cannot rule out that these dwarf galaxies have dark haloes with constant density cores, we find that their rotation curves are consistent with cold dark matter haloes as expected in a $\Lambda$ CDM cosmology.

\section{THE DATA}

The HI rotation curves that we use in this paper have been derived from the data presented in Swaters (1999, hereafter S99). The HI observations were done with the Westerbork Synthesis Radio Telescope (WSRT) as part of the Westerbork HI Survey of Spiral and Irregular Galaxies Project (WHISP, see Kamphuis, Sijbring \& van Albada 1996). The observations and data reduction are discussed in detail in S99. From the sample of 73 late-type dwarf galaxies we only selected those galaxies that according to S99 have high quality rotation curves (no strong asymmetries and high signalto-noise ratio). This sample consists of 20 galaxies, which have inclination angles in the range $39^{\circ} \leq i \leq 80^{\circ}$. Galaxies with $i>80^{\circ}$ have been excluded from the sample because they require a somewhat different analysis.

Table 1 lists the properties of the galaxies in our sample. The absolute magnitudes, disk scale lengths, and central surface brightnesses have been determined from $R$-band photometry (Swaters \& Balcells 2001, hereafter SB01). The distances are as adopted by SB01: where possible stellar distance indicators have been used, mostly brightest stars. If these were not available, distances based on group membership were used, or, if these were unavailable as well, the distance was calculated from the HI systematic velocity following the prescription in Kraan-Korteweg (1986) with an adopted Hubble constant of $H_{0}=75 \mathrm{~km} \mathrm{~s}^{-1} \mathrm{Mpc}^{-1} . B-R$ colors, available for only 6 galaxies, are also taken from SB01.

The original HI observations have been obtained with a typical beam of $14^{\prime \prime} \times 14^{\prime \prime} / \sin \delta$ (with $\delta$ the object's declination). In general, the signal-to-noise ratio at this resolution was too low to obtain reliable rotation curves. Therefore, the data were convolved to a resolution of approximately $30^{\prime \prime} \times 30^{\prime \prime}$. Velocity fields were constructed by fitting Gaussian curves to the observed line profiles at each position. Next, the rotation velocities and their formal errors were determined by fitting a tilted-ring model to the velocity fields assuming constant inclination and position angles. Where possible the orientation angles were determined from the velocity fields, and in the remaining cases from the optical images. For details about the determination of the tilted ring parameters see S99. Note, however, that our analysis is different from the one presented in S99, where an iterative method, based on modelling of the observed data cubes, was used to approximately correct the rotation curve for the effects of beam smearing. The rotation curves we use here, however, have not been corrected for beam smearing. In- 
Table 1. Properties of sample of late-type dwarf galaxies.

$\begin{array}{rrrccccccc}\text { UGC } & D & M_{R} & \mu_{0}^{R} & R_{d} & V_{\text {last }} & N_{V} & B-R & i & \Lambda \text { CDM } \\ 731 & 8.0 & -16.63 & 23.0 & 1.65 & 74 & 12 & 0.85 & 57 & + \\ 3371 & 12.8 & -17.74 & 23.3 & 3.09 & 86 & 11 & 1.08 & 49 & + \\ 4325 & 10.1 & -18.10 & 21.6 & 1.63 & 92 & 8 & 0.85 & 41 & + \\ 4499 & 13.0 & -17.78 & 21.5 & 1.49 & 74 & 9 & -- & 50 & + \\ 5414 & 10.0 & -17.55 & 21.8 & 1.49 & 61 & 7 & -- & 55 & ? \\ 6446 & 12.0 & -18.35 & 21.4 & 1.87 & 80 & 11 & -- & 52 & + \\ 7232 & 3.5 & -15.31 & 20.2 & 0.33 & 44 & 5 & 0.81 & 59 & ? \\ 7323 & 8.1 & -18.90 & 21.2 & 2.20 & 86 & 10 & -- & 47 & ? \\ 7399 & 8.4 & -17.12 & 20.7 & 0.79 & 109 & 12 & 0.78 & 55 & + \\ 7524 & 3.5 & -18.14 & 22.2 & 2.58 & 79 & 31 & -- & 46 & + \\ 7559 & 3.2 & -13.66 & 23.8 & 0.67 & 33 & 10 & -- & 61 & ? \\ 7577 & 3.5 & -15.62 & 22.5 & 0.84 & 18 & 10 & -- & 63 & ? \\ 7603 & 6.8 & -16.88 & 20.8 & 0.90 & 64 & 12 & -- & 78 & ? \\ 8490 & 4.9 & -17.28 & 20.5 & 0.66 & 78 & 30 & -- & 50 & + \\ 9211 & 12.6 & -16.21 & 22.6 & 1.32 & 65 & 10 & -- & 44 & + \\ 11707 & 15.9 & -18.60 & 23.1 & 4.30 & 100 & 13 & -- & 68 & + \\ 11861 & 25.1 & -20.79 & 21.4 & 6.06 & 153 & 10 & -- & 50 & + \\ 12060 & 15.7 & -17.95 & 21.6 & 1.76 & 74 & 9 & -- & 40 & + \\ 12632 & 6.9 & -17.14 & 23.5 & 2.57 & 76 & 17 & 0.91 & 46 & + \\ 12732 & 13.2 & -18.01 & 22.4 & 2.21 & 98 & 15 & -- & 39 & +\end{array}$

Column (1) lists the UGC number of the galaxy. Columns (2) - (6) list the distance to the galaxy (in $\mathrm{Mpc}$ ), absolute $R$-band magnitude, central $R$-band surface brightness (in mag $\operatorname{arcsec}^{-2}$ ), scale length of the stellar disk (in kpc), and the observed rotation velocity $V_{\text {last }}\left(\right.$ in $\mathrm{km} \mathrm{s}^{-1}$ ) at the last measured point. Column (7) lists the number of data points, $N_{V}$, along the rotation curve (two data points per beam). Columns (8) and (9) list the $B-R$ color (if available) and the adopted inclination angle (in degrees), respectively. Magnitudes and central surface brightnesses have been corrected for inclination and galactic extinction, but not for internal extinction. Finally, column (10) indicates whether the galaxy is consistent with a $\Lambda \mathrm{CDM}$ cosmology $(+)$ or whether no meaningful fit can be found (?). See the discussion in $\S$ for details.

stead, we beam-smear our models before comparison with the data, following the procedure detailed in $\S 3.2$.

\section{ROTATION CURVE FITTING}

\subsection{Mass components}

For the mass modelling presented in this paper, we assume that there are three main mass components in each galaxy: an infinitesimally thin gas disk, a thick stellar disk, and a spherical dark halo. We closely follow the procedure outlined in van den Bosch et al. (2000; hereafter BRDB), which we briefly outline below for completeness.

In order to determine the contribution of the gas to the galaxy's circular velocity, we make the assumption that the gas is distributed axisymmetrically in an infinitesimally thin disk. Under this assumption the circular velocities due to the self-gravity of the gas can be computed from the gas surface density using equation [2-146] of Binney \& Tremaine (1987). We model the HI density distribution as follows:

$\Sigma_{\mathrm{HI}}(R)=\Sigma_{0}\left(\frac{R}{R_{1}}\right)^{\beta} \mathrm{e}^{-R / R_{1}}+f \Sigma_{0} \mathrm{e}^{-\left(\left(R-R_{2}\right) / \sigma\right)^{2}}$.

The first term is identical to the surface density profile used in BRDB, and represents an exponential disk with scale length $R_{1}$ and with a central hole, the extent of which depends on $\beta$. The second term corresponds to a Gaussian ring with radius $R_{2}$ and a FWHM $\propto \sigma$. The flux ratio between these two components is set by $f$. The form of equa- tion (1) has no particular physical motivation, but should be regarded as a fitting function. When computing the circular velocities of the atomic gas, we multiply $\Sigma_{\mathrm{HI}}$ by a factor 1.3 to correct for the contribution of helium.

For the stellar disk we assume a thick exponential

$\rho^{*}(R, z)=\rho_{0}^{*} \exp \left(-R / R_{d}\right) \operatorname{sech}^{2}\left(z / z_{0}\right)$

where $R_{d}$ is the scale length of the disk. Throughout we set $z_{0}=R_{d} / 6$. The exact value of this ratio, however, does not significantly influence the results. The circular velocity of the stellar disk is computed using equation [A.17] in Casertano (1983), and properly scaled with the stellar $R$-band massto-light ratio $\Upsilon_{R}$. None of the galaxies in our sample has a significant bulge component.

We assume that initially the dark and baryonic matter virialize to form a spherical halo with a density distribution given by

$\rho(r)=\frac{\rho_{0}}{\left(r / r_{s}\right)^{\alpha}\left(1+r / r_{s}\right)^{3-\alpha}}$,

with $r_{s}$ being the scale radius of the halo, such that $\rho \propto r^{-\alpha}$ for $r \ll r_{s}$ and $\rho \propto r^{-3}$ for $r \gg r_{s}$. For $\alpha=1$ equation (3) reduces to the NFW profile (Navarro, Frenk \& White 1997). We define the concentration parameter $c=r_{200} / r_{s}$, with $r_{200}$ the radius inside of which the mean density is 200 times the critical density for closure, i.e.,

$\frac{r_{200}}{h^{-1} \mathrm{kpc}}=\frac{V_{200}}{\mathrm{~km} \mathrm{~s}^{-1}}$. 
Here $V_{200}$ is the circular velocity at $r_{200}$, and $h=$ $H_{0} / 100 \mathrm{~km} \mathrm{~s}^{-1} \mathrm{Mpc}^{-1}$.

The formation of the stellar and gaseous disks due to the cooling of the baryons inside the virialized halo leads to a contraction of the dark matter component. We make the assumption that the baryonic collapse is slow, and take this adiabatic contraction of the dark halo into account following the procedure in Barnes \& White (1984), Blumenthal et al. (1986) and Flores et al. (1993). The halo mass inside radius $r$, required for the adiabatic contraction computations, is given by

$M_{\text {halo }}(r)=M_{200} \frac{\mu(x c)}{\mu(c)}$

with $x=r / r_{200}$ and

$\mu(x)=\int_{0}^{x} y^{2-\alpha}(1+y)^{\alpha-3} \mathrm{~d} y$

\subsection{Beam smearing}

As is evident from the results presented in S99 and BRDB, it is important that the effects of beam-smearing are properly taken into account. Rather than attempting to deconvolve the observations (which is an ill-defined problem), we convolve our models with the effective point spread function $P$ (i.e., the beam) of the interferometer. The convolved surface brightness at a position $(x, y)$ on the plane of the sky is

$\tilde{\Sigma}(x, y)=\int_{0}^{\infty} \mathrm{d} r r \int_{0}^{2 \pi} \mathrm{d} \theta \Sigma\left(r^{\prime}\right) P\left(r, \theta-\theta_{0}\right)$.

Here $r^{\prime}=\sqrt{x^{\prime 2}+y^{\prime 2}}$, where $x^{\prime}=x+r \cos \theta$ and $y^{\prime}=$ $(y+r \sin \theta) / \cos i$ are the Cartesian coordinates in the equatorial plane of the disk, $i$ is the disk's inclination angle, and $\theta_{0}$ is the angle between the major axes of the galaxy and the beam (for which we adopt a two-dimensional Gaussian, see BRDB). The underlying surface brightness, $\Sigma(R)$, is modeled by equation (1). Note that we assume that the HI distribution is axisymmetric.

Beam smearing also affects the observed rotation velocities $\tilde{V}_{\text {rot }}$ at a position $(x, y)$ on the plane of the sky by causing gas from a larger area of the disk to contribute to the observed line of sight velocity:

$\tilde{V}_{\text {rot }}(x, y)=\frac{1}{\tilde{\Sigma}} \int_{0}^{\infty} \mathrm{d} r r \int_{0}^{2 \pi} \mathrm{d} \theta \Sigma\left(r^{\prime}\right) V_{\operatorname{los}}\left(x^{\prime}, y^{\prime}\right) P\left(r, \theta-\theta_{0}\right)$

where $V_{\text {los }}$ is the line of sight velocity. Throughout we assume that the gas moves on circular orbits in the plane of the disk and has zero intrinsic velocity dispersion. As argued by Swaters (1999), the asymmetric drift corrections as calculated from the HI distribution are generally small and have little effect on the derived rotation curves.

\subsection{Fitting procedure}

The first step in fitting mass models to the rotation curves is to determine the best-fit model for the true underlying
HI distribution. The surface density distribution of equation (11) has six free parameters. Note, however, that $\Sigma_{0}$ is completely determined by normalizing the models to the total mass in HI and can thus be ignored in the fitting routine. We determine the best-fit parameters by minimizing

$\chi_{\mathrm{HI}}^{2}=\sum_{i=1}^{N_{\mathrm{HI}}}\left(\frac{\Sigma_{\mathrm{obs}}\left(R_{i}\right)-\tilde{\Sigma}\left(R_{i}\right)}{\Delta \Sigma_{\mathrm{obs}}\left(R_{i}\right)}\right)^{2}$,

with $\Sigma_{\text {obs }}$ the observed HI density distribution at $30^{\prime \prime}$ resolution and $\Delta \Sigma_{\text {obs }}$ the corresponding errors. The results are shown in the upper-right panels of Figures A1 A19. Open circles correspond to the observed HI surface density and solid lines to the best-fit model. In most cases, the unsmeared HI distribution of the best-fit models, indicated by dashed lines, is fairly similar to that after convolution with the beam, indicating that the effects of beam-smearing for these data are only small. In two cases, UGC 7524 and UGC 7603, our fitting function (equation [1]) can not satisfactorily describe the data. In these cases we use the data of the full resolution (see $\S$ ) as a model for the true underlying HI distribution.

Once $\Sigma_{\mathrm{HI}}$ is known we can compute the beam-smeared model rotation curves. For a given choice of the Hubble constant the mass models described above have four free parameters to fit the data: $\Upsilon_{R}, \alpha, c$, and $V_{200}$ (or equivalently $r_{200}$ ). For a given $\left(\alpha, \Upsilon_{R}\right)$ we determine the best-fitting $c$ and $V_{200}$ by minimizing

$\chi_{\mathrm{vel}}^{2}=\sum_{i=1}^{N_{\mathrm{vel}}}\left(\frac{V_{\mathrm{obs}}\left(R_{i}\right)-\tilde{V}\left(R_{i}\right)}{\Delta V_{\mathrm{obs}}\left(R_{i}\right)}\right)^{2}$.

Here $\Delta V_{\text {obs }}$ are the formal errors on $V_{\text {obs }}$ from the tilted-ring model fits, and $\tilde{V}\left(R_{i}\right)$ is computed from equation (\$) with $x=R_{i}$ and $y=0$.

\subsection{Uncertainties on the rotation velocities}

An important issue in constraining the density distribution of the dark matter haloes is how to interpret $\chi_{\mathrm{vel}}^{2}$. One can only use the absolute values of $\chi_{\mathrm{vel}}^{2}$ to compute confidence levels for our models, if the errors $\Delta V_{\text {obs }}$ are the proper, normally distributed errors, there are no systematic errors, the data points are independent, the assumptions underlying the model are correct, and the mass-model is a proper representation of the real mass distribution. However, the fact that the minimum $\chi_{\mathrm{vel}}^{2}$ differs considerably from the number of degrees of freedom for almost all galaxies indicates that we do not meet these criteria. This does not come as a surprise. First of all, errors in the assumed inclination angle, distance, beam parameters, and the distribution of gas and stars all lead to systematic errors in the dark matter density distribution. Furthermore, there are numerous assumptions underlying our mass-models, each of which may be in error. For instance, we assume that the halo is sperical, that the disk is axisymmetric and that the gas moves on perfectly circular orbits with zero intrinsic velocity dispersion (i.e., we thus ignore asymmetric drift). In addition, we assume that $\Sigma_{\text {gas }}=1.3 \Sigma_{\mathrm{HI}}$ and that $\Upsilon_{R}$ is constant throughout the stellar disk. We thus ignore any contribution from molecular and/or ionized gas as well as any radial changes in stellar population. Given all these potential sources of confusion, 
we only use $\chi_{\text {vel }}^{2}$ to assess the relative quality of the model fits. We do not try to assign any confidence levels to the absolute values of either $\chi_{\text {vel }}^{2}$ or $\Delta \chi_{\text {vel }}^{2}$. Henceforth, if, for instance, a model for a particular galaxy yields a smaller $\chi_{\mathrm{vel}}^{2}$ for $\alpha=0$ than for $\alpha=1$, the model with a constant density core provides a better fit than the NFW model, but it does not necessarily imply that the rotation curve is inconsistent with CDM. For these reasons, we use physical criteria (discussed in $\S$. rather than criteria based on uncertain confidence levels to assess whether a model is consistent with the data.

\subsection{Degeneracies in the mass modelling}

Before interpreting the results in terms of constraints on the density distribution of dark matter haloes it is useful to examine some models. To that end, we construct three model galaxies moulded after UGC 731, i.e., the models have the same HI and stellar disks as UGC 731, and we adopt the same distance and inclination angle (see Table $\mathbb{1}$ ). We add a dark matter halo with a density distribution given by equation (3) and compute the beam-smeared rotation velocities at $15^{\prime \prime}$ intervals. This corresponds to roughly half the beam size and is identical to the interval between actual data points of the rotation curve of UGC 731. We convolve the velocity field using the same beam-size and beam orientation as for the true UGC 731 data. Finally, we add a random Gaussian error to the model rotation velocities (with variance $\Delta V)$. All three models have the same mass distribution: $\alpha=1, c=20, V_{200}=75 \mathrm{~km} \mathrm{~s}^{-1}$, and $\Upsilon_{R}=2.0(\mathrm{M} / \mathrm{L})_{\odot}$. What we vary, however, is the way the rotation curve is sampled: Model 1 has a rotation curve with 6 data points extending out to $0.06 r_{200}$ and with $\Delta V=2.0 \mathrm{~km} \mathrm{~s}^{-1}$. The rotation curve of model 2 has twice as many data points, thus extending twice as far out, and has the same $\Delta V$. Model 3, finally, has a rotation curve that extends equally far as that of model 2, but with $\Delta V=0.2 \mathrm{~km} \mathrm{~s}^{-1}$. For comparison, the real data of UGC 731 consists of 12 data points with $\langle\Delta V\rangle=1.4 \mathrm{~km} \mathrm{~s}^{-1}$, and model 2 is thus a fair representation of the actual data. Also, as can be seen in column (7) in Table 1, the number of data points for the other galaxies in our sample varies from 5 (UGC 7232) to 31 (UGC 7524). Note that, because of distance effects, a larger $N_{V}$ does not necessarily mean that the density distribution of the halo is probed to larger radii.

We analyze our model rotation curves in the same way as we analyze the data for the dwarf galaxies. The results are shown in Figure 1. A number of general trends, which are also present in the real data (see Figures A1 A19), are immediately apparent. First of all, the halo concentration $c$ of the best-fit model decreases with increasing $\Upsilon_{R}$ and $\alpha$. This is easily understood in terms of the total enclosed mass which has to be similar for different models. Secondly, there is an $\alpha_{\text {crit }}$ for which $V_{200}$ is maximal and $c=1$. For $\alpha>\alpha_{\text {crit }}$ the best fitting halo concentration $c<1$, implying that the scale radius $r_{s}$ is larger than the virial radius $r_{200}$. Clearly, for $c<1$, equation (3) no longer is an appropriate description of the dark matter distribution, and we therefore demand that $c \geq 1$. Consequently, for $\alpha>\alpha_{\text {crit }}$ the halo concentration $c=1$ and the quality of the fits rapildy decreases (i.e., $\chi^{2}$ increases).

In addition to these trends, a large amount of non- uniqueness is apparent, which can be associated with two distinct degeneracies. First of all, for any given cusp slope $\alpha$, the relative amount of mass in the stellar disk can be traded off against the amount of mass in the halo, while maintaining virtually equally good fits to the data, i.e., different combinations of $\left(\Upsilon_{R}, c, V_{200}\right)$ yield similar values of $\chi_{\mathrm{vel}}^{2}$. This degeneracy is well-known from the rotation curves of high surface brightness galaxies (e.g., van Albada et al. 1985), and is generally referred to as the mass-to-light ratio degeneracy. The second degeneracy is that for a given mass-to-light ratio $\Upsilon_{R}$, different combinations of $\left(\alpha, c, V_{200}\right)$ with $\alpha \lesssim 1.5$ yield virtually equally good fits to the data, unless the errors on the observed rotation velocities are sufficiently small (cf. models 2 and 3). This degeneracy, which we refer to as the cusp-core degeneracy, is a consequence of the fact that rotation curves only sample a small fraction of the dark matter density distribution (see also Lake \& Feinswog 1989).

To illustrate the nature of this cusp-core degeneracy we construct the circular velocity curve, $V_{\alpha=1}(r)$, of a dark matter halo with $\alpha=1, c=25$, and $V_{200}=100 \mathrm{kms}^{-1}$. Next, for a range of values for $\alpha$, we seek the values of $c$ and $V_{200}$ for which $V_{\alpha}(r)$ (with $\alpha \neq 1.0$ ) best fits $V_{\alpha=1}(r)$ out to a certain radius $r_{\max }$. The results are shown in Figure 2 for $r_{\max }=0.15 r_{200}$ (indicated by a dotted vertical line). The thick curve in the upper panels corresponds to $V_{\alpha=1}(r)$, normalized to $V_{200}$. The thin curves correspond to the bestfitting $V_{\alpha}(r)$ for $\alpha=0.0,0.2,0.4, \ldots, 1.8$. As is evident from the left panels in Figure 2, where we plot the circular velocities only out to $0.2 r_{200}$, the different $V_{\alpha}(r)$ curves are in fact very similar. Only for $\alpha \gtrsim 1.5$ does $V_{\alpha}(r)$ start to deviate more significantly from $V_{\alpha=1}(r)$. This explains why the reduced $\chi_{\text {vel }}^{2}$ of models 1 and 2 increases strongly for $\alpha \gtrsim 1.5$. For $\alpha \lesssim 1.5$ the circular velocity curves out to $r_{\max }$ are remarkably similar, and only very accurate rotation curves (i.e., with small $\Delta V$ ) can discriminate between the different curves (cf. models 2 and 3). Alternatively, accurate constraints on the actual density distribution of the dark matter requires a rotation curve that either extends sufficiently far, or that has sufficient independent measurements at very small radii. This is evident from the lower two panels of Figure 2, which plot the normalized difference $\left(V_{\alpha}-V_{\alpha=1}\right) / V_{200}$ as function of the normalized radius, and which show how the different $V_{\alpha}$ curves diverge for both $r / r_{200} \gtrsim 0.2$ and $r / r_{200} \lesssim 0.02$. Unfortunately, in practice HI rotation curves rarely extend to large enough radii, do not have enough spatial resolution, have too large errors, and suffer too much from systematic effects to lift this cusp-core degeneracy.

\section{RESULTS}

The results for each individual galaxy are presented in Figures A1 A19 and discussed in some detail in the Appendix. For UGC 7557 no converging fit to the observed rotation curve could be achieved with the mass-model described in Section 3 , and therefore no results are plotted for this galaxy (see discussion in the Appendix). The four left panels of Figures A1 A19 show (from top to bottom) $\chi_{\text {red }}^{2}, c, V_{200}$, and the corresponding baryon fraction $f_{\text {bar }}$ (see $\S 3.5$ ), all as a function of the cusp slope $\alpha$, and for three different values of $\Upsilon_{R}$. Here $\chi_{\text {red }}^{2} \equiv \chi_{\text {vel }}^{2} / N_{\mathrm{df}}$, with $N_{\mathrm{df}}$ the number of de- 


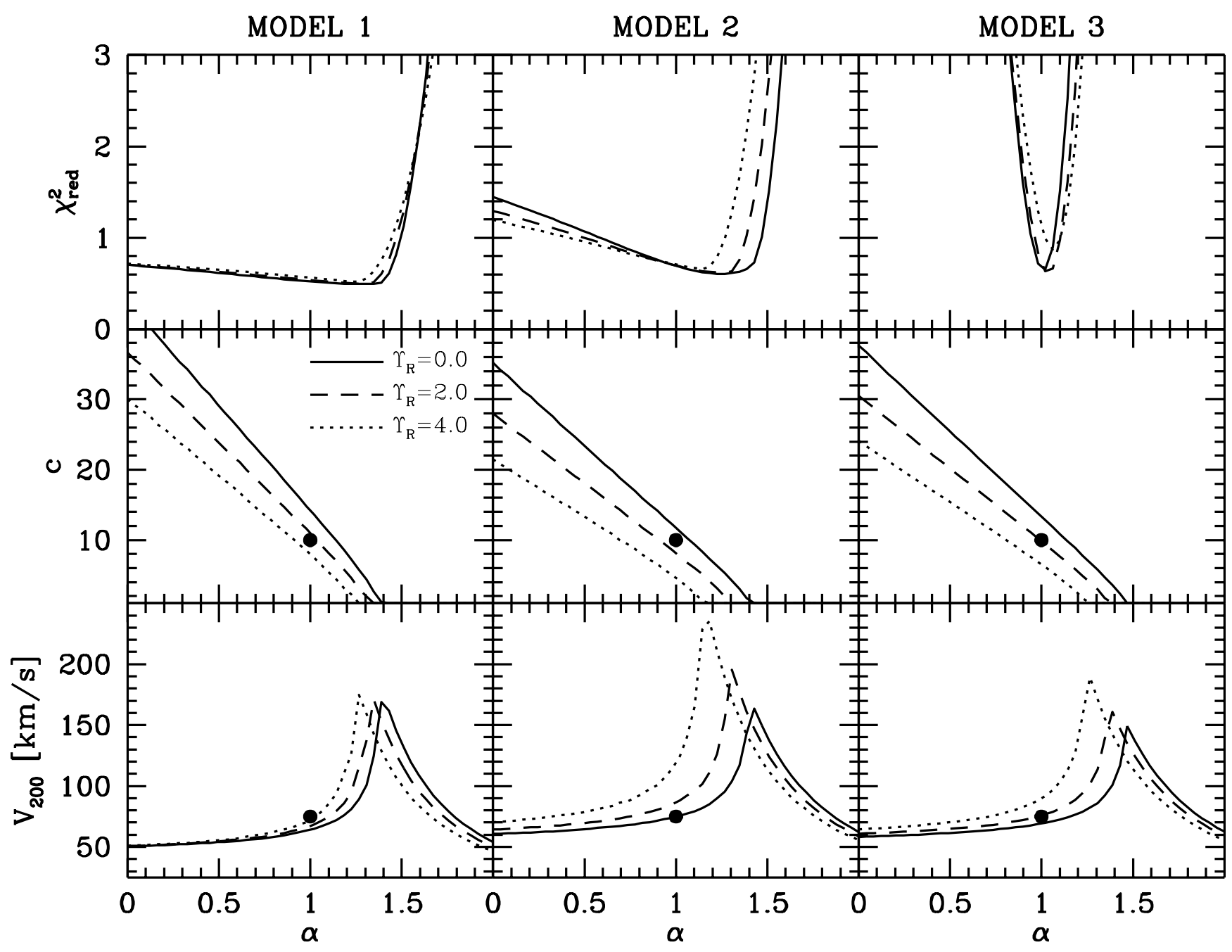

Figure 1. Results of analyzing three model rotation curves, moulded after UGC 731. All models have the same density distribution (indicated by a black dot); they only differ in the extent of the rotation curve and the errors on the rotation velocities, as indicated in the text. In fitting the model rotation curves three different mass-to-light ratios have been assumed: $\Upsilon_{R}=0, \Upsilon_{R}=2.0(\mathrm{M} / \mathrm{L}) \odot($ which is the input value of the models), and $\Upsilon_{R}=4.0(\mathrm{M} / \mathrm{L})_{\odot}$. The labeling is as indicated in the left middle panel. Note that the mass models derived from the rotation curves of models 1 and 2 (both with $\Delta V=2 \mathrm{~km} \mathrm{~s}^{-1}$ ) are strongly degenerate. The rotation curve of model 3, for which $\Delta V=0.2 \mathrm{~km} \mathrm{~s}^{-1}$, however, allows a fairly accurate recovery of the input mass model, although the mass-to-light ratio degeneracy remains.

grees of freedom. The same general trends we found for the model galaxies are also apparent in the data, i.e., the halo concentration $c$ decreases with increasing $\Upsilon_{R}$ and $\alpha$, and above a certain value of $\alpha$ the quality of the fits decreases rapidly while $c=1$. As we indicated in $\S$ we have used data that is convolved to a lower resolution in order to enhance the signal-to-noise ratio. We have checked that our best fit models are consistent with the higher resolution data and found good agreement.

As we have argued in $\S 3.4$ above, we can not simply use $\chi_{\text {red }}^{2}$ to put confidence levels on the various models. Furthermore, we have pointed out that two distinct degeneracies hamper a unique mass-decomposition, which is readily apparent from the fact that models with very different cusp slopes and/or mass-to-light ratios yield roughly equally good fits (see for instance UGC 11707, UGC 12060, and UGC 12632). However, some constraints can be imposed by only considering models that are physically re- alistic. For instance, models with $\Upsilon_{R}=0$ are unrealistic, and are therefore not considered meaningful model fits. In addition, we can use the baryon fraction of each model to check its physical validity. For each model we compute the baryonic mass fraction $f_{\text {bar }} \equiv\left(M_{\text {gas }}+M_{\text {stars }}\right) / M_{200}$ with $M_{200}=r_{200} V_{200}^{2} / G$ the total mass of the galaxy (baryons plus dark matter). For currently popular cosmologies with $\Omega_{0}=0.3$ and $h=0.7$, and using recent Big Bang nucleosynthesis constraints on the baryon density $\left(\Omega_{\mathrm{bar}}=0.02 h^{-2}\right.$; Burles \& Tytler 1998), one expects a universal baryons fraction of roughly 0.14 . Note that because we ignore any molecular and/or ionized gas, and because feedback may drive galactic winds and expel baryons from the halo, $f_{\text {bar }}$ may be significantly lower than the universal value. In order to leave some room for the uncertainties in the cosmological parameters, in what follows we shall only consider models unrealistic if $f_{\text {bar }}>0.2$. In section 5 below we address the 


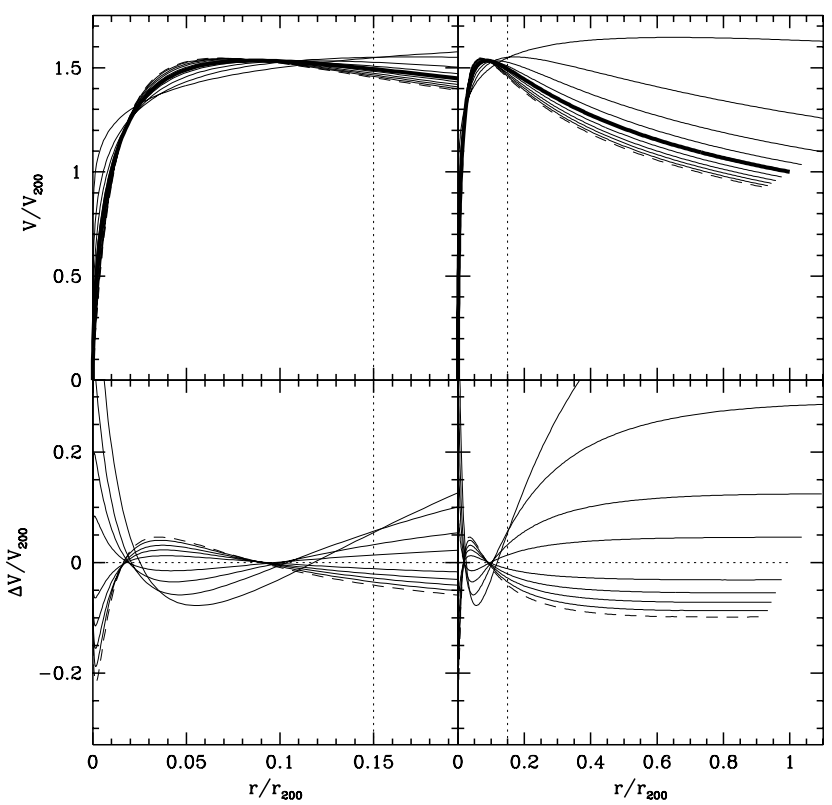

Figure 2. An illustration of the cusp-core degeneracy. The thick solid lines in the upper two panels correspond to the circular velocity of a halo with a density distribution of equation (3) with $\alpha=1.0$ and $c=25$. The abscissa and ordinate are normalized to $r_{200}$ and $V_{200}$ of this density distribution, respectively, The thin lines are best-fits to the inner parts of this circular velocity curve of models with $\alpha=0.0,0.2,0.4, \ldots, 1.8$, once again normalized to $r_{200}$ and $V_{200}$ of the $\alpha=1.0$ model. For clarity, the model with $\alpha=0.0$ is indicated by dashed lines. The lower panels plot the normalized differences, $\left(V_{\alpha}-V_{\alpha=1}\right) / V_{200}$ as function of $r / r_{200}$. When fitting the models, only the velocities out to $r_{\max }=0.15 r_{200}$ (indicated by vertical dotted lines) are taken into account.

effects a possible lower bound on $f_{\text {bar }}$ might have on our conclusions.

We can put some further constraints on the mass models by considering what range of $R$-band mass-to-light ratios to expect. Using the Bruzual \& Charlot (1993) stellar population models we have computed $B-R$ and $\Upsilon_{R}$ for a Scalo (1986) IMF and two different star formation histories. Figure 3 plots $\Upsilon_{R}$ versus $B-R$ for three different metallicities and for both a constant star formation rate (left panel) and a single burst stellar population (right panel). For the six galaxies in our sample for which SB01 obtained $B$-band photometry we find $\langle B-R\rangle=0.87 \pm 0.09$ (see Table 11). If we assume that there is no internal extinction in these galaxies, this implies $0.5 \lesssim \Upsilon_{R} \lesssim 1.1$ for the stellar population models investigated here. This ignores the contribution of any non-luminous baryonic component that may have the same radial distribution as the stars, and which would increase the mass-to-light ratio. In Table 2 we list the parameters of the best-fit models with $\alpha=1$ for both $\Upsilon_{R}=0$ and $\Upsilon_{R}=1.0(\mathrm{M} / \mathrm{L})_{\odot}$. Although models with $\Upsilon_{R}=0$ are unrealistic, these best-fit models yield a useful upper limit on $c$ (cf. Pickering et al. 1997; Navarro 1998). The models with $\Upsilon_{R}=1.0(\mathrm{M} / \mathrm{L}) \odot$ are chosen to represent a typical mass-to-light ratio. Furthermore, a comparison of two models with different mass-to-light ratios sheds light on the (non)-uniqueness of the mass models.

The panels in the lower-right corners of Figures A1.
Table 2. Best-fit parameters for models with $\alpha=1$.

\begin{tabular}{|c|c|c|c|c|c|c|c|}
\hline UGC & $\Upsilon_{R}$ & $c$ & $V_{200}$ & $f_{\mathrm{bar}}$ & $c_{\min }$ & $\langle c\rangle$ & $c_{\max }$ \\
\hline \multirow[t]{2}{*}{731} & 0.0 & 16.0 & 51.3 & $2.3 \times 10^{-2}$ & 4.7 & 15.6 & 31.3 \\
\hline & 1.0 & 13.5 & 52.3 & $2.8 \times 10^{-2}$ & 4.7 & 15.6 & 31.1 \\
\hline \multirow[t]{2}{*}{3371} & 0.0 & 9.5 & 68.6 & $1.6 \times 10^{-2}$ & 4.4 & 14.6 & 29.2 \\
\hline & 1.0 & 8.0 & 69.8 & $2.1 \times 10^{-2}$ & 4.4 & 14.5 & 29.1 \\
\hline \multirow[t]{2}{*}{4325} & 0.0 & 30.9 & 53.5 & $2.1 \times 10^{-2}$ & 4.6 & 15.5 & 30.9 \\
\hline & 1.0 & 25.7 & 52.7 & $4.1 \times 10^{-2}$ & 4.6 & 15.5 & 30.9 \\
\hline \multirow[t]{2}{*}{4499} & 0.0 & 9.0 & 58.1 & $2.7 \times 10^{-2}$ & 4.6 & 15.2 & 30.4 \\
\hline & 1.0 & 1.6 & 131.5 & $3.7 \times 10^{-3}$ & 3.7 & 12.3 & 24.7 \\
\hline \multirow[t]{2}{*}{5414} & 0.0 & $<1.0$ & 253.6 & $1.8 \times 10^{-4}$ & 3.0 & 10.1 & 20.2 \\
\hline & 1.0 & $<1.0$ & 128.8 & $2.3 \times 10^{-3}$ & 3.7 & 12.4 & 24.9 \\
\hline \multirow[t]{2}{*}{6446} & 0.0 & 17.4 & 52.0 & $4.1 \times 10^{-2}$ & 4.7 & 15.6 & 31.1 \\
\hline & 1.0 & 7.0 & 59.5 & $4.8 \times 10^{-2}$ & 4.5 & 15.1 & 30.1 \\
\hline \multirow[t]{2}{*}{7232} & 0.0 & 4.2 & 116.0 & $2.3 \times 10^{-4}$ & 3.8 & 12.8 & 25.5 \\
\hline & 1.0 & $<1.0$ & 59.6 & $3.8 \times 10^{-3}$ & 4.5 & 15.1 & 30.1 \\
\hline \multirow[t]{2}{*}{7323} & 0.0 & 4.7 & 129.3 & $1.4 \times 10^{-3}$ & 3.7 & 12.4 & 24.9 \\
\hline & 1.0 & $<1.0$ & 193.9 & $1.5 \times 10^{-3}$ & 3.3 & 11.0 & 22.0 \\
\hline \multirow[t]{2}{*}{7399} & 0.0 & 23.1 & 62.8 & $1.3 \times 10^{-2}$ & 4.5 & 14.9 & 29.8 \\
\hline & 1.0 & 15.2 & 70.9 & $1.4 \times 10^{-2}$ & 4.4 & 14.5 & 29.0 \\
\hline \multirow[t]{2}{*}{7524} & 0.0 & 8.5 & 71.9 & $1.1 \times 10^{-2}$ & 4.3 & 14.4 & 28.9 \\
\hline & 1.0 & 4.8 & 82.5 & $1.4 \times 10^{-2}$ & 4.2 & 13.9 & 27.9 \\
\hline \multirow[t]{2}{*}{7559} & 0.0 & 1.4 & 135.4 & $1.4 \times 10^{-4}$ & 3.7 & 12.2 & 24.4 \\
\hline & 1.0 & 1.2 & 135.1 & $1.6 \times 10^{-4}$ & 3.7 & 12.2 & 24.4 \\
\hline \multirow[t]{2}{*}{7603} & 0.0 & 5.5 & 87.9 & $3.0 \times 10^{-3}$ & 4.1 & 13.7 & 27.4 \\
\hline & 1.0 & $<1.0$ & 217.3 & $3.9 \times 10^{-4}$ & 3.2 & 10.6 & 21.2 \\
\hline \multirow[t]{2}{*}{8490} & 0.0 & 24.2 & 50.3 & $3.3 \times 10^{-2}$ & 4.7 & 15.6 & 31.3 \\
\hline & 1.0 & 13.5 & 57.2 & $2.9 \times 10^{-2}$ & 4.6 & 15.2 & 30.5 \\
\hline \multirow[t]{2}{*}{9211} & 0.0 & 18.3 & 43.6 & $5.6 \times 10^{-2}$ & 4.9 & 16.2 & 32.4 \\
\hline & 1.0 & 14.8 & 44.5 & $6.1 \times 10^{-2}$ & 4.8 & 16.1 & 32.2 \\
\hline \multirow[t]{2}{*}{11707} & 0.0 & 13.1 & 67.3 & $5.3 \times 10^{-2}$ & 4.4 & 14.7 & 29.3 \\
\hline & 1.0 & 11.2 & 66.9 & $7.1 \times 10^{-2}$ & 4.4 & 14.7 & 29.3 \\
\hline \multirow[t]{2}{*}{11861} & 0.0 & 16.0 & 106.3 & $2.6 \times 10^{-2}$ & 3.9 & 13.1 & 26.1 \\
\hline & 1.0 & 12.5 & 100.6 & $7.6 \times 10^{-2}$ & 4.0 & 13.2 & 26.4 \\
\hline \multirow[t]{2}{*}{12060} & 0.0 & 34.6 & 46.6 & $7.8 \times 10^{-2}$ & 4.8 & 16.0 & 31.9 \\
\hline & 1.0 & 24.3 & 46.5 & $1.1 \times 10^{-1}$ & 4.8 & 16.0 & 31.9 \\
\hline \multirow[t]{2}{*}{12632} & 0.0 & 14.0 & 51.6 & $2.8 \times 10^{-2}$ & 4.7 & 15.6 & 31.1 \\
\hline & 1.0 & 12.6 & 51.4 & $3.7 \times 10^{-2}$ & 4.7 & 15.6 & 31.1 \\
\hline \multirow[t]{2}{*}{12732} & 0.0 & 10.3 & 67.6 & $5.3 \times 10^{-2}$ & 4.4 & 14.7 & 29.3 \\
\hline & 1.0 & 6.8 & 73.3 & $4.7 \times 10^{-2}$ & 4.3 & 14.3 & 28.7 \\
\hline
\end{tabular}

For each galaxy we list the parameters of two best-fit models with $\alpha=1$ (i.e., a dark matter halo with a $r^{-1}$ density cusp): one with $\Upsilon_{R}=0$ and the other with $\Upsilon_{R}=1.0(\mathrm{M} / \mathrm{L})_{\odot}$. In addition to the best-fit parameters $c, V_{200}$ (in $\mathrm{km} \mathrm{s}^{-1}$ ), and the resulting baryon fraction $f_{\mathrm{bar}}$, we list the mean halo concentration, $\langle c\rangle$, for $\Lambda \mathrm{CDM}$ haloes with the same $V_{200}$ as the best-fit model, as well as the $2 \sigma$ lower and upper limits ( $c_{\min }$ and $c_{\max }$, respectively) of the distribution of $c$. These values are computed using the model of Bullock et al. (1999) for the $\Lambda$ CDM cosmology used here. Consistency with $\Lambda \mathrm{CDM}$ requires that $c_{\min }<c<c_{\max }$ and $f_{\mathrm{bar}}<0.2$.

A19, plot the observed rotation curves (open circles with errorbars) together with four models with $\Upsilon_{R}=1.0(\mathrm{M} / \mathrm{L})_{\odot}$. These are the best-fit models for $\alpha=0$ (solid lines), $\alpha=$ 0.5 (dotted lines), $\alpha=1.0$ (short-dash lines), and $\alpha=1.5$ (long-dash lines). These plots illustrate the typical quality of the model fits and the dependence on the cusp slope $\alpha$. In most cases the individual curves for the four models can hardly been discerned, further emphasizing the cusp-core degeneracy discussed above. 

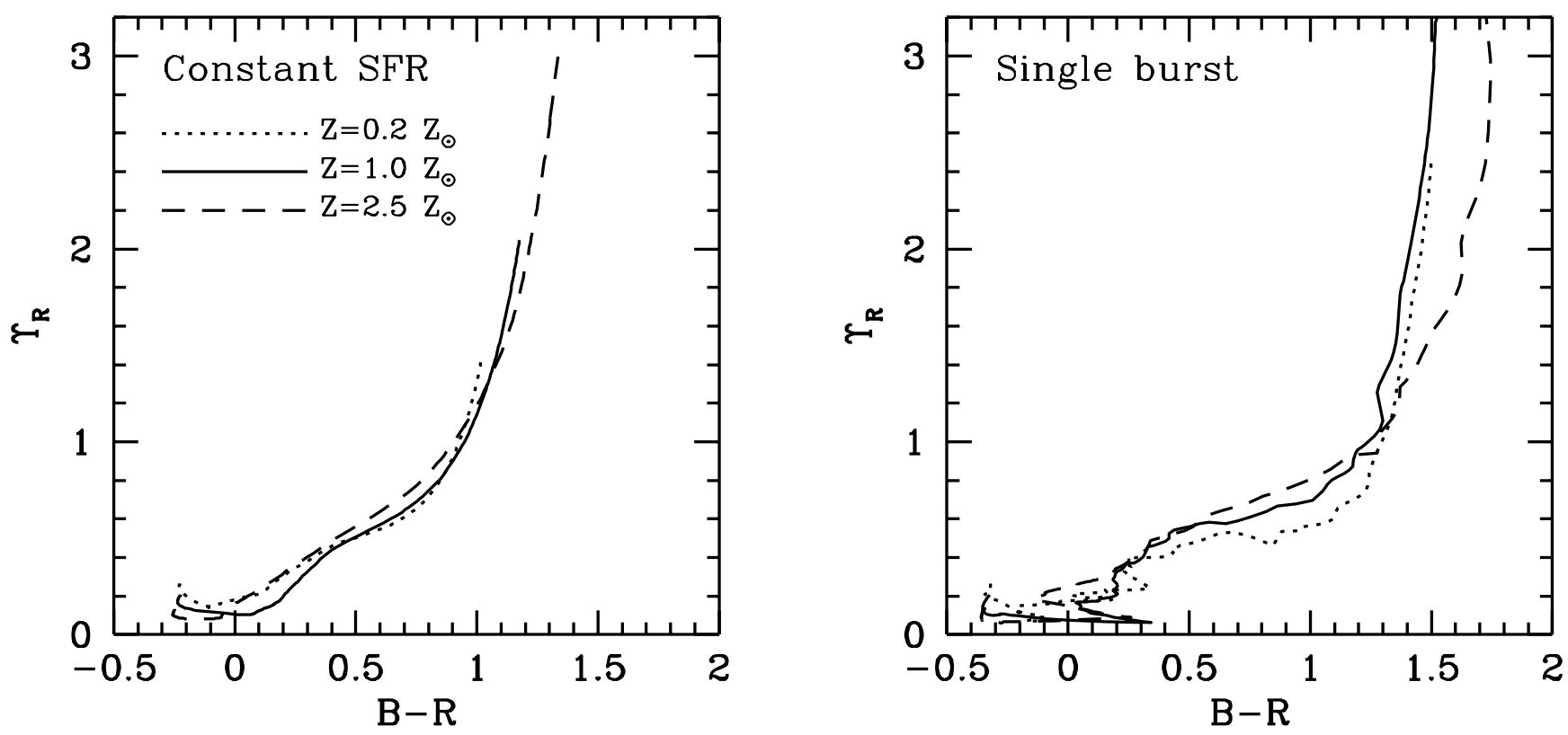

Figure 3. The $R$-band mass-to-light ratio $\Upsilon_{R}$ as function of the $B-R$ color of Bruzual \& Charlot (1993) stellar population models with a Scalo IMF and either a constant star formation rate (left panel) or a single burst of star formation (right panel). Results are shown for three different metallicities: one fifth Solar (dotted lines), Solar (solid lines) and two and half times Solar (dashed lines). For the dwarf galaxies in our sample with known $B-R$ we find $\langle B-R\rangle=0.87 \pm 0.09$, which implies that $\Upsilon_{R} \simeq 0.5-1.1(\mathrm{M} / \mathrm{L}) \odot$.

\section{IMPLICATIONS FOR THE NATURE OF THE DARK MATTER}

The main goal of this paper is to assess whether or not the rotation curves of dwarf galaxies are consistent with CDM. Before we can address this, we need to define what "consistent with CDM" means in the context of the density distribution of dark haloes. Ideally, one would like to place confidence levels on whether or not a rotation curve is consistent with certain CDM predictions. However, as we discussed in $\S 3.4$, we can not use our $\chi^{2}$ statistic to compute such confidence levels. Therefore we follow a different approach. Several studies that have claimed inconsistencies between (dwarf) galaxy rotation curves and CDM haloes, indicated that when a model with $\alpha=1.0$ is fitted the implied halo concentration $c$ is too low to be consistent with CDM (i.e., Pickering et al. 1997; Navarro 1998). We follow this approach and investigate whether the distribution of $c$ for our best-fitting models with $\alpha=1.0$ is consistent with predictions for a particular CDM model.

High resolution $N$-body simulations have shown that haloes virialize to density distributions of the form of equation (3) with $\alpha \sim 1$. Different simulations, however, often yield different values for the concentrations. Furthermore, the distribution of halo concentrations is fairly broad, and its median depends on the mass of the halo, its redshift, and the cosmology (Cole \& Lacey 1996; Navarro, Frank \& White 1996, 1997; Avila-Reese et al. 1999; Bullock et al. 1999; Jing 2000; Jing \& Suto 2000). Henceforth, there is (currently) no well-defined boundary for $c$ to be considered "consistent with CDM". Instead, one can only ask whether the statistical properties of a sample of rotation curves are consistent with CDM for a given cosmology and according to a given set of simulations.

In what follows we focus on the currently popular
$\Lambda \mathrm{CDM}$ cosmology with $\Omega_{0}=0.3, \Omega_{\Lambda}=0.7, h=0.7$, and $\sigma_{8}=1.0$. The particular simulations to which we compare our results are presented in Bullock et al. (1999; hereafter B99), who also give a simple recipe for computing $c$ and its scatter as function of halo mass 1 . The reason for using this particular recipe is that it is tested against a large statistical sample of several thousand haloes. The expected value of $c$ for each galaxy (as determined by $V_{200}$ of the best-fit model), and the $2 \sigma$ deviations from the median, indicated by $c_{\min }$ and $c_{\max }$, are listed in Table 2 .

We now define a rotation curve to be consistent with $\Lambda \mathrm{CDM}$ if for $\alpha=1.0$ and $0.5 \leq \Upsilon_{R} \leq 1.1$ there is a best-fit model with $c_{\min } \leq c \leq c_{\max }$ and $f_{\mathrm{bar}} \leq 0.2$. These limits on $\Upsilon_{R}$ and $f_{\text {bar }}$ are motivated in $\S 4$. Note that we do not demand that the models with $\alpha=1.0$ yield the best fit (i.e., minimum $\chi_{\text {red }}^{2}$ ) of all models. Instead, we demand that there is a model with $\alpha=1.0$ for which the resulting parameters are realistic. According to this definition, we find that 14 out of the 20 dwarf galaxies in our sample are consistent with $\Lambda \mathrm{CDM}$. These galaxies are indicated by a ' + ' in column (10) in Table 1 .

But what about the other 6 galaxies? For UGC 7577 no converging fit could be obtained at all, and this galaxy is discarded from the following discussion. The best fit models of the other five galaxies all have $\alpha=0.0$ (constant density core) and $\Upsilon_{R}=0$ (which is unphysical). In Figure 1 we plot $f_{\text {bar }}$ and $V_{200}$ for these five galaxies as functions of $\Upsilon_{R}$, whereby $\alpha$ is kept constant at zero. As can be seen, the inferred values of $f_{\text {bar }}$ rapidly decrease with increasing $\Upsilon_{R}$, while $V_{200}$ rapidly increases. For $\Upsilon_{R} \gtrsim 0.5 \mathrm{M}_{\odot} / \mathrm{L}_{\odot}$, which

$\dagger$ This model uses somewhat different definitions for the halo mass and concentration, which we convert to the $c$ and $M_{200}$ used in our analysis. 

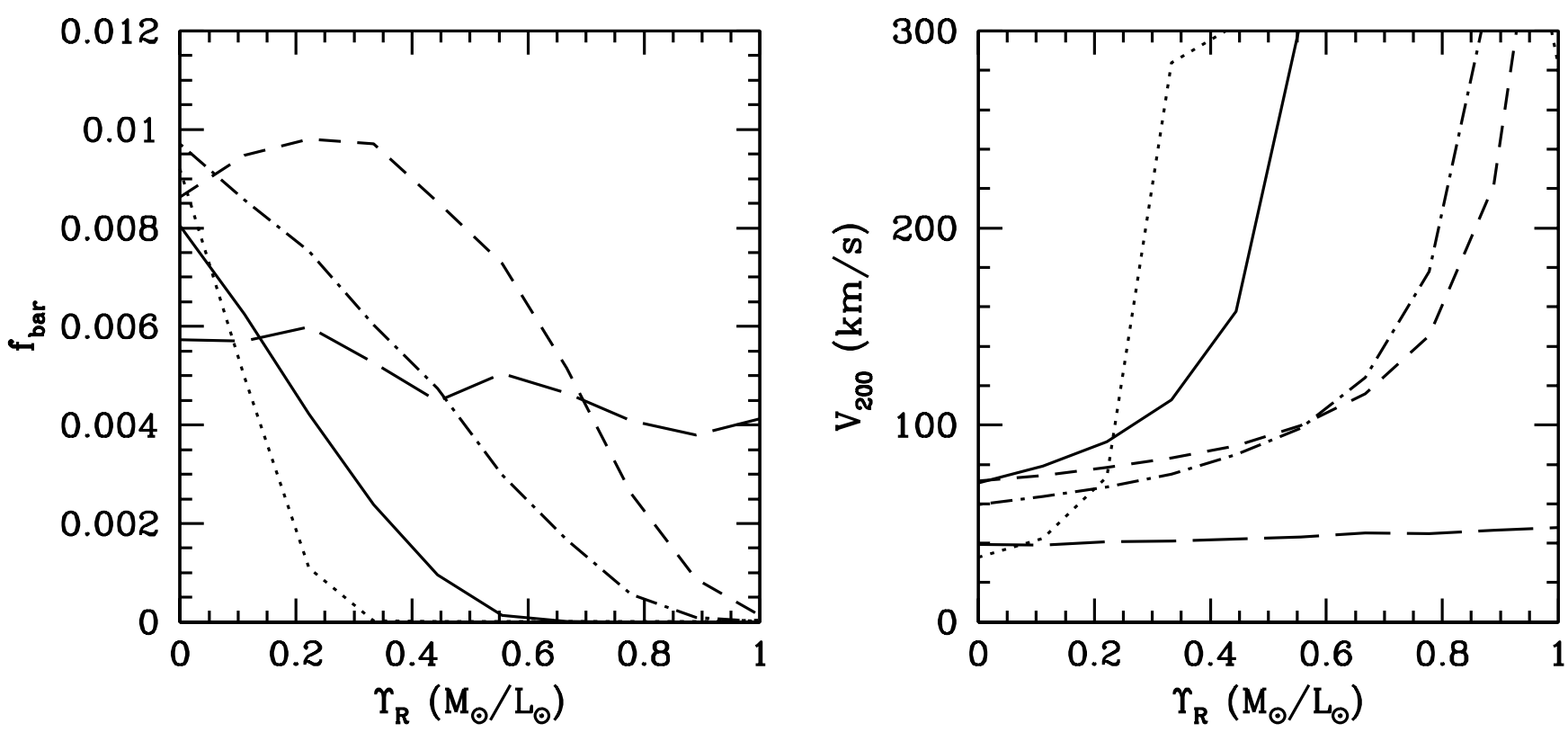

Figure 4. The baryon fractions (left) and virial velocities (right) as function of $\Upsilon_{R}$ for the five galaxies that are inconsistent with $\Lambda$ CDM haloes: UGC 5414 (solid lines), UGC 7232 (dotted lines), UGC 7323 (short-dashed lines), UGC 7559 (long-dashed lines) and UGC 7603 (dot-dashed lines). The results plotted here are for $\alpha=0$ (haloes with constant density cores) only. Note how $f_{\text {bar }}$ deceases rapidly with increasing $\Upsilon_{R}$, while $V_{200}$ rapidly increases. For $\Upsilon_{R}>0.5 \mathrm{M}_{\odot} / \mathrm{L}_{\odot}$ (which is the more realistic regime), $f_{\text {bar }}<8 \times 10^{-3}$, while $V_{200}>90 \mathrm{~km} \mathrm{~s}^{-1}$ (except for UGC 7559). Given our current understanding of feedback, such low baryon fractions are hard to reconcile with the large halo masses, and we therefore consider these rotation curve fits unrealistic (see discussion in text).

is the more realistic regime, one finds values of $f_{\text {bar }}$ well below 0.01. With a universal baryon fraction of $\sim 0.14$ this implies that $>90$ percent of the available baryons would have to be expelled from the disk. Although current understanding of feedback is very limited, and we are reluctant to impose stringent lower limits on $f_{\text {bar }}$, such high ejection efficiencies seem inconsistent with the high values of $V_{200}$ inferred from the best-fit models (see e.g., Dekel \& Silk 1986; Efstathiou 2000). In particular, recent hydro-dynamical simulations have indicated that even starburst driven winds are extremely inefficient in expelling matter from systems with such high virial velocities (Mac Low \& Ferrara 1999; Strickland \& Stevens 2000). Thus, unless a mechanism can be devised that can expell $\gtrsim 90$ percent of the available baryons from haloes with $V_{200} \gtrsim 90 \mathrm{~km} \mathrm{~s}^{-1}$, we conclude that for these five galaxies none of the $\left(\alpha, \Upsilon_{R}\right)$-models are realistic. This implies that whereas the rotation curves of these galaxies are inconsistent with the $\Lambda \mathrm{CDM}$ model, they do not support an alternative picture in which, for instance, dark matter haloes have constant density cores. For these galaxies either (1) our mass-model is inadequate, (2) there are systematic errors in the data, or (3) one or more of the assumptions listed in $\S 3.5$ are wrong.

The good agreement between CDM predictions and the majority of the rotation curves analyzed here is also illustrated in Figure $\mathrm{B}$ where we plot $c$ as function of $V_{200}$ for the best-fit models with $\alpha=0$ (left panels), $\alpha=1.0$ (middle panels; see also Table ), and $\alpha=1.5$ (right panels). Results are plotted for both $\Upsilon_{R}=0$ (upper panels) and $\Upsilon_{R}=1.0(\mathrm{M} / \mathrm{L}) \odot$ (lower panels). Solid circles correspond to galaxies that are consistent with $\Lambda$ CDM, open circles to galaxies for which no meaningful fit can be obtained. The solid and dashed lines in the middle panels indicate the mean and the $2 \sigma$ intervals of the predictions based on the B99 model. For comparison, we also plot (dotted lines) the predictions based on the model of Navarro, Frenk \& White (1997) For $\alpha=0.0$ and $\alpha=1.5$ no such lines are plotted, since no model predictions exist for these cases. As already noted by Eke, Navarro \& Steinmetz (2000), the B99 model predicts concentration values a factor $\sim 1.6$ larger at $V_{200}=30 \mathrm{~km} \mathrm{~s}^{-1}$, and a slightly steeper mass dependence. Since the disagreement between the two models is relatively small compared to the expected scatter in $c$, our conclusions are not sensitive to the fact that we focus our discussion on the B99 model. It is apparent that for all galaxies for which a meaningful fit is obtained, the best fit values for $c$ and $V_{200}$ are consistent with the expected values in a $\Lambda \mathrm{CDM}$ cosmology.

In total we thus find that 14 out of 20 galaxies are consistent with CDM. For the remaining 6 galaxies no meaningful fit to the observed rotation curves can be obtained with our mass models for any value of $\alpha$. Henceforth, these galaxies are neither consistent with CDM, nor with any other viable alternative. We thus conclude that at present there is no convincing evidence that dwarf galaxies (or low surface brightness galaxies, see BRDB) have dark matter haloes that are inconsistent with CDM. However, we wish to point out that this conclusion is based on the presumption that $\mathrm{CDM}$ haloes have $\alpha \simeq 1$.0. If future simulations confirm the results by Fukushige \& Makino (1997), Moore et al. (1998) and Klypin et al. (2000), that CDM produces more steeply cusped dark matter haloes with $\alpha \simeq 1.5$, we

$\ddagger$ Computed using the procedure outlined in their Appendix with $f=0.01$ and $C=3.41 \times 10^{3}$ 


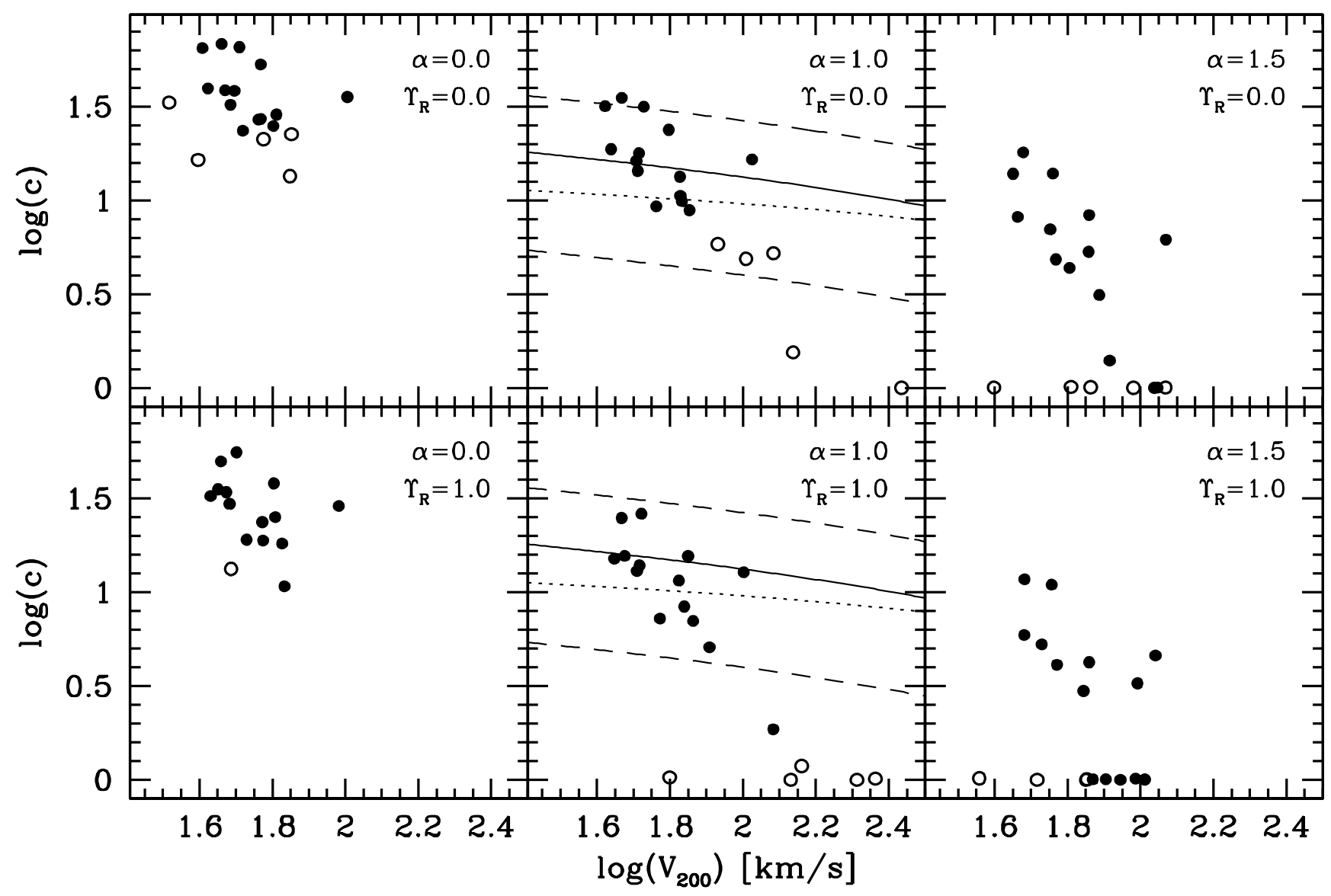

Figure 5. The logarithm of the best-fit concentration parameter as function of the logarithm of $V_{200}$ of the best-fit model. Results are shown for two different stellar mass-to-light ratios and three different values of $\alpha$ (as indicated in the panels). Solid circles correspond to galaxies that are consistent with the $\Lambda \mathrm{CDM}$ model, whereas open circles indicate galaxies for which no meaningful fit can be obtained (indicated by ' + ' and '?' in Table $\mathbb{1}$, respectively). The solid and dashed lines in the middle panels indicate the mean and the $2 \sigma$ limits of the distribution of halo concentrations as predicted by the B99 model for the $\Lambda$ CDM cosmology. All galaxies for which the best-fit models are physically realistic are consistent with this model. However, for $\alpha=1.5$ (right panels) it is apparent that a significant fraction of the best-fit models are unrealistic in that they have $c=1$ ). Henceforth, if future simulations confirm that CDM yields haloes with $\alpha \simeq 1.5$ rather than $\alpha \simeq 1.0$, the rotation curves analyzed here may signal a true problem for the CDM paradigm.

would have to conclude that the rotation curves analyzed here are only marginally consistent with CDM. This is evident from the plots in Figures A1 A19 and Figure , which show that for $\alpha \gtrsim 1.5$ the fits in general become rather poor, and often unrealistic. Furthermore, we have made the assumption, based on stellar population models, that $\Upsilon_{R} \lesssim 1.1(\mathrm{M} / \mathrm{L}) \odot$. If, however, the true mass-to-light ratio of the stellar disks is significantly higher and/or dwarf galaxies have large amounts of (centrally concentrated) molecular gas, some of the galaxies analyzed here will have dark matter haloes that are inconsistent with CDM.

Although we have shown that the majority of the rotation curves in our sample are consistent with CDM, it does not imply that they are inconsistent with any of CDM's alternatives, such as WDM, SFDM or SIDM. In fact, in some cases models with a constant density core provide better fits to the rotation curves than for $\alpha=1.0$ (e.g., UGC 7524 and UGC 9211). Ultimately, one might hope to use the rotation curves of (dwarf) galaxies to put some constraints on the central phase space densities or core radii of their dark matter haloes. This in turn constrains the masses and/or interaction cross sections of the dark matter particles. Unfortunately, the rotation curves analyzed here do not put any significant constraints on the actual nature of the dark matter: they are consistent with any dark matter species that yields haloes with $0 \lesssim \alpha \lesssim 1.5$. In order for the rotation curves to put stringent constraints on the nature of the dark matter, we have to be able to much better constrain the density distribution of the dark matter haloes. However, given the numerous potential sources of systematic errors and both the mass-to-light ratio degeneracy and the cuspcore degeneracy discussed above it seems unlikely that HI rotation curves alone will be able to provide any significant constraints on the nature of the dark matter.

\subsection{Comparison with previous work}

Our conclusion that the rotation curves of dwarf galaxies are consistent with a $\Lambda \mathrm{CDM}$ cosmology is at odds with previous studies (Moore 1994; Navarro et al. 1996; Moore et al. 1999b; Blais-Ouellette, Amram \& Carignan 2000). There are several reasons that may contribute to this discrepancy. First of all, 
the previous studies have mostly used the same, small sample of dwarf galaxies, the rotation curves of which have been determined in different ways. We have used high-resolution HI rotation curves for a new, relatively large sample of dwarf galaxies for which the rotation curves have been derived in a uniform way. Furtermore, some of the dwarf galaxies in the previous studies have high inclinations or strong non-circular motions e.g., as the result of a bar. Rotation curves derived from such galaxies are likely to suffer from systematic effects. For this reason, we excluded galaxies with strong bars and inclinations larger than $80^{\circ}$. In addition, we have improved upon previous studies by taking beam-smearing and adiabatic contraction into account. As beam-smearing can mimic the presence of a constant density core, it is imperative that these effects are properly accounted for (see e.g., BRDB). Also, we have stressed the importance of degeneracies, systematic errors, and the fact that not all models are physically meaningful. Finally, some of the studies that have argued against NFW haloes fitted their models in some ad hoc manner to the last measured data point or the maximum of the rotation curve. However, there is no reason for treating a certain data point in a special way. In our analysis we fitted the models to all data points, weighted by their errors.

\section{SUMMARY}

We have analyzed high resolution HI rotation curves for a sample of 20 late-type dwarf galaxies. Taking beamsmearing and adiabatic contraction into account we have investigated to what extent these rotation curves put constraints on the (central) density distributions of dark matter haloes.

We have shown that two distinct degeneracies hamper a unique mass-decomposition. The first one, which we call the cusp-core degeneracy, owes to the fact that the observed rotation curves in general only sample the circular velocities of the system at intermediate radii. No data is available at either very small or very large radii, making it virtually impossible to discriminate between haloes with a constant density core and a $r^{-1}$ cusp. The other degeneracy, the mass-to-light ratio degeneracy, is well known from the rotation curves of (high surface brightness) spiral galaxies: as a result of the uncertainty in the stellar mass-to-light ratio, the relative amount of mass in the stellar disk can be exchanged with the amount of dark matter. It is noteworthy that several studies in the past have suggested that dwarf galaxies are not impeded by this mass-to-light ratio degeneracy, and are therefore ideally suited to infer constraints on dark matter haloes. However, when the effects of beam smearing are taken into account, late-type dwarf galaxies are also plagued by the mass-to-light ratio degeneracy (see also the discussion in S99).

In $\sim 70$ percent of the cases analyzed here, we find that the rotation curves are consistent with a $\Lambda$ CDM cosmology. In the remaining $\sim 30$ percent, no meaningful fit to the observed rotation curves could be obtained with our mass models for any value of the inner slope of the halo density profile. Thus, although these galaxies are inconsistent with the $\Lambda$ CDM model, they can not be considered to support any of the alternative dark matter models (unless a mechanism can be devised that can explain extremely small baryonic mass fractions). This is most likely due to systematic errors and/or the fact that some of the assumptions underlying the models are incorrect. This emphasizes that care is to be taken when interpreting rotation curve fits; sometimes inconsistencies with CDM predictions are claimed without exploring the full freedom in halo parameters, and without addressing whether or not alternative models (i.e., with a constant density core) can yield realistic fits to the data.

Our main conclusion, therefore, is that there is no convincing evidence against dark matter haloes of dwarf galaxies having $r^{-1}$ cusps. The HI rotation curves analyzed here are consistent with dark matter haloes with $\alpha=1$ and with concentrations as predicted for the currently popular $\Lambda \mathrm{CDM}$ cosmology. Together with the results for LSB galaxies presented in BRDB and S99, we thus conclude that, based on the rotation curves of galaxies, there is currently no need to abandon the idea that dark matter is cold and collisionless. However, if future high resolution simulations confirm earlier findings of cusp slopes in the range of $\alpha=1.5$, or if it turns out that dwarf galaxies have disks with $\Upsilon_{R} \gg 1.0(\mathrm{M} / \mathrm{L})_{\odot}$, it may be necessary to abandon CDM in favor of an alternative that yields haloes that are less steeply cusped.

It is important to point out that the rotation curves studied here are also consistent with the presence of dark matter haloes with constant density cores. Thus, although current data does not require abandoning CDM, neither does it allow us to rule against its alternatives such as WDM, SFDM, or SIDM. Discriminating between these various dark matter models requires rotation curves of extremely high accuracy. Given the numerous sources for (systematic) errors, and the typical beam size of radio observations, we conclude that based on HI rotation curves alone at best weak limits on cosmological parameters and/or the nature of the dark matter can be obtained. In order to place more stringent constraints on the actual density distribution of dark matter haloes one needs either data of higher spatial resolution, such as obtainable with $\mathrm{H} \alpha$ spectroscopy (i.e., Courteau 1997; Blais-Ouellette, Carignan \& Amram 1999; Swaters et al. 2000; Blais-Ouelette et al. 2000) or CO observations (e.g., Sofue et al. 1999), and one needs to obtain independent constraints on the disk's mass-to-light ratio from for instance stellar velocity dispersions (Bottema 1993) or, in the case of barred disk galaxies, from a detailed modelling of the velocity fields (e.g., Englmaier \& Gerhard 1999; Weiner, Sellwood \& Williams 2001).

\section{ACKNOWLEDGMENTS}

We are grateful to Julianne Dalcanton for reading an earlier version of the paper, to Stéphane Charlot for providing results from his stellar population models, and to the referee for his insightful comments. This research was supported in part by the National Science Foundation under Grant No. PHY94-07194. FvdB was supported by NASA through Hubble Fellowship grant \# HF-01102.11-97.A awarded by the Space Telescope Science Institute, which is operated by AURA for NASA under contract NAS 5-26555. 


\section{REFERENCES}

Avila-Reese V., Firmani C., Klypin A.A., Kravtsov A.V., 1999, MNRAS, 310, 527

Barnes J.E., White S.D.M., 1984, MNRAS, 211, 753

Barnes J.E., Hernquist L., 1992, Nature, 360, 715

Bento M.C., Bertolami O.. Rosenfeld R., Teodoro L., 2000, preprint astro-ph/0003350)

Binney J.J., Tremaine S.D., 1987, Galactic Dynamics. (Princeton: Princeton University Press)

Binney J.J., Gerhard O.E., Silk J., 2001, MNRAS, 321, 471

Blais-Ouellette S., Carignan C., Amram P., 1999, in ASP Conf. Ser. 182, Galaxy Dynamics, ed. D. Merritt, M. Valluri \& J. A. Sellwood (San Francisco: ASP), 387

Blais-Ouellette S., Amram P., Carignan C., 2000, preprint (astro$\mathrm{ph} /$ 006449)

Blumenthal G.R., Faber S.M., Flores R., Primack J.R., 1986, ApJ, 301, 27

Bottema R., 1993, A\&A, 275, 16

Bruzual G.A., Charlot S., 1993, ApJ, 405, 538

Bullock J.S., Kolatt T.S., Sigad Y., Somerville R.S., Kravtsov A.V.. Klypin A.A., Primack J.R., Dekel A., 1999, preprint astro-ph/9908159

Bullock J.S., Kravtsov A.V., Weinberg D.H., 2000, preprint astro-ph/0002214, B99

Burles S., Tytler D., 1998, Sp. Sci. Rev., 84, 65

Burkert A., 1995, ApJ, 447, L25

Burkert A., Silk J., 1997, ApJ, 488, L55

Burkert A., 2000, ApJ, 534, L143

Carlson E.D., Machacek M.E., Hall L.J., 1992, ApJ, 398, 43

Casertano S., 1983, MNRAS, 203, 735

Cole S., Lacey S., 1996, MNRAS, 281, 716

Colín P., Avila-Reese V., Valenzuela O., 2000, ApJ, 542, 622 astro-ph/0004115)

Courteau S., 1997, AJ, 114, 2402

Dalcanton J.J., Bernstein R.A., 2000, in XVth IAP Meeting Dynamics of Galaxies: From the Early Universe to the Present, ed. F. Combes, G. A. Mamom, \& V. Charmandaris, ASP Conf. Ser. 197, p. 161

Dalcanton J.J., Hogan C.J., 2000, preprint astro-ph/0004381)

Dekel A., Silk J., 1986, ApJ, 303, 39

Efstathiou G., 2000, MNRAS, 317, 697

Eke V.R. Navarro J.F., Steinmetz M., 2000, preprint astro$\mathrm{ph} /$ 012337)

Englmaier P., Gerhard O.E., 1999, MNRAS, 304, 512

Firmani C., D'Onghia E., Avila-Reese V., Chincarini G., Hernández X., 2000, MNRAS, 315, L29

Firmani C., D’Onghia E., Chincarini G., Hernández X., AvilaReese V., 2001, MNRAS, 321, 713

Flores R., Primack J.R., Blumenthal G.R., Faber S.M., 1993, ApJ, 412, 443

Flores R., Primack J.R., 1994, ApJ, 427, L1

Fukushige T., Makino J., 1997, ApJ, 477, L9

Gelato S., Sommer-Larson J., 1999, MNRAS, 303, 321

Ghigna S., Moore B., Governato F., Lake G., Quinn T., Stadel J., 1998, MNRAS, 300, 146

Goodman J., 2000, New Astron. 5. 103

Hannestad S., 1999, preprint (astro-ph/9912558)

Hogan C.J., Dalcanton J.J., 2000, preprint (astro-ph/0002330)

Hu W., Peebles P.J.E., 1999, preprint (astro-ph/9910222

Hunter D.A., Wilcots E.M., van Woerden H., Callagher J.S., Kohle S., 1998, ApJ, 495, L47

Jing Y.P., 2000, ApJ, 535, 30

Jing Y.P., Suto Y., 2000, ApJ,529, L69

Kamionkowski M., Liddle A.R., 2000, Phys. Rev. Let. 84, 4525

Kamphuis J.J., Sijbring D., van Albada T.S., 1996, A\&AS, 116, 15

Kaplinghat M., Knox L., Turner M.S., 2000, Phys. Rev. Let. 85,
3335

Kauffmann G., White S.D.M., Guiderdoni B., 1993, MNRAS, 264 201

Kochanek C.S., White M., 2000, ApJ, 543, 514

Klypin A.A., Gottlöber S., Kravtsov A.V., Khokholov A.M., 1999a, ApJ, 516, 530

Klypin A.A., Kravtsov A.V., Valenzuela O., Prada F., 1999b, ApJ, 522, 82

Klypin A.A., Kravtsov A.V., Bullock J.S., Primack J.R., 2000, preprint (astro-ph/0006343)

Kraan-Korteweg R.C., 1986, A\&AS, 66, 255

Lake G., Feinswog L., 1989, AJ, 98, 166

Mac Low M.-M., Ferrrara A., 1999, ApJ, 513, 142

Matos T., Siddharta G.F., Urena-López L.A., 2000, Clas. Quantum Grav. 17, 1707

McGaugh S.S., de Blok W.J.G., 1998, ApJ, 499, 41

Miralde-Escude J., 2000, preprint astro-ph/0002013)

Mohapatra R.N., Teplitz V.L., 2000, preprint astro-ph/0001362

Moore B., 1994, Nature, 370, 629

Moore B., Governato F., Quinn T., Stadel J., Lake G., 1998, ApJ, 499, L5

Moore B., Ghigna S., Governato F., Lake G., Quinn T., Stadel J., Tozzi P., 1999a, ApJ, 524, L19

Moore B., Quinn T., Governato F., Stadel J., Lake G., 1999b, MNRAS, 310, 1147

Moore B., Gelato S., Jenkins A., Pearce F.R., Quilis V., 2000, ApJ, 535, L21

Navarro J.F., 1998, preprint astro-ph/9807084

Navarro J.F., Eke V.R., Frenk C.S., 1996, MNRAS, 283, 72

Navarro J.F., Frenk C.S., White S.D.M., 1996, ApJ, 462, 563

Navarro J.F., Frenk C.S., White S.D.M., 1997, ApJ, 490, 493

Peebles P.J.E., Vilenkin A., 1999, Phys. Rev. D., 60, 103506

Peebles P.J.E., 2000, ApJ, 534, L127

Pickering T.E., Impey C.D., van Gorkom J.H., Bothun G.D., 1997, AJ, 114, 1858

Scalo J.N., 1986, Fundam. Cosmic Phys., 11, 1

Sellwood J.A., 2000, ApJ, 540, L1

Sofue Y., Tutui Y., Honma M., Tomita A.. Takamiya T., Koda J., Takeda Y., 1999, preprint (astro-ph/9905056)

Sommer-Larsen J., Dolgov A., 1999, preprint astro-ph/9912166 Spergel D.N., Steinhardt P.J., 2000, Phys. Rev. Let. 84, 17

Stil J., 1999, PhD Thesis, Leiden University

Strickland D.K., Stevens I.R., 2000, MNRAS, 314, 511

Swaters R.A., 1999, PhD Thesis, University of Groningen (S99)

Swaters R.A., Madore B.A., Trewhella M., 2000, ApJ, 531, L107

Swaters R.A., Balcells M., 2001, in preparation (SB01)

van Albada T.S., Bahcall J.N., Begeman K., Sancisi R., 1985, ApJ, 295, 305

van den Bosch F.C., Robertson B.E., Dalcanton J.J., de Blok W.J.G., 2000, AJ, 119, 1579 (BRDB)

Weiner B.J., Sellwood J.A., Williams T.B., 2001, ApJ, 546, 931

White S.D.M., Springel V., 1999, in The First Stars, Proc MPA/ESO workshop, ed. A. Weiss et al., astro-ph/9911378

White M., Croft R.A., 2000, ApJ, 539, 497

Yoshida N., Springel V., White S.D.M., Tormen G., 2000, ApJ, 535, L103

\section{APPENDIX A: COMMENTS ON INDIVIDUAL GALAXIES}

UGC 731: As for the models, which were moulded after this galaxy (see $\S 3.5$ ), there is a large degeneracy in the model parameters. The only robust results seem to be that $\alpha \lesssim 1.6$ and $f_{\text {bar }} \lesssim 0.05$. Most importantly, the observed rotation velocities of this galaxy are in excellent agreement with CDM haloes, i.e., for $\alpha=1$, we find $c \simeq 16-2.3 \Upsilon_{R}$. 
UGC 3371: The best-fit models prefer a dark matter halo with a steep central cusp. However, for a given $\Upsilon_{R}$ the minimum $\chi^{2}$ is achieved for $\alpha=\alpha_{\text {crit }}$, and these bestfit models are therefore unrealistic. Although $\chi^{2}$ seems to depend rather strongly on $\alpha$, this owes mainly to the extremely small errorbars on $V_{\text {rot }}$ : the best-fit rotation curves with $\Upsilon_{R}=1.0(\mathrm{M} / \mathrm{L}) \odot$ and $\alpha=0,0.5$, and 1.0 can hardly be discerned by eye. For $\alpha=1$ we find $c \simeq 9.5-1.45 \Upsilon_{R}$, and we thus conclude that UGC 3371 is consistent with CDM.

UGC 4325: The quality of the fit improves considerably with increasing mass-to-light ratio up to $\Upsilon_{R} \simeq$ $7.5(\mathrm{M} / \mathrm{L})_{\odot}$, after which $\chi^{2}$ increases rapidly. However, for $\Upsilon_{R} \gtrsim 7(\mathrm{M} / \mathrm{L})_{\odot}$ the best-fit models have $c=1$ and are thus unrealistic. Furthermore, S99 has shown that $0.5 \lesssim$ $\Upsilon_{R} \lesssim 2.0$ based on the stellar velocity dispersions, and $\Upsilon_{R} \gtrsim 3(\mathrm{M} / \mathrm{L}) \odot$ is unlikely in the light of stellar population models and UGC 4325's color of $B-R=0.85$ (see Figure B). Clearly, the best-fit model is not the most realistic model. As is evident from the lower-left panel, models with $\Upsilon_{R}=1.0(\mathrm{M} / \mathrm{L}) \odot$ provide reasonable fits to the data, virtually independent of $\alpha$. For $\alpha=1$ we find $c=30.9-4.8 \Upsilon_{R}$, and we thus conclude that UGC 4325 is consistent with CDM.

UGC 4499: The quality of the fit depends strongly on the stellar mass-to-light ratio. For $\Upsilon_{R} \gtrsim 2.0(\mathrm{M} / \mathrm{L}) \odot$, the models become unrealistic. The best fitting models have $\Upsilon_{R}=0.0$, which is also unphysical. For $\Upsilon_{R}=1(\mathrm{M} / \mathrm{L})_{\odot}$, the best-fit models with $\alpha=0.0,0.5$, and 1.0 yield virtually equally good fits to the data, but models with $\alpha \gtrsim 1.1$ are excluded by the data, since they require $c<1$. For $\alpha=1$ we find $c \simeq 9.0-7.0 \Upsilon_{R}$. Henceforth, for $\Upsilon_{R}=1.0(\mathrm{M} / \mathrm{L}) \odot$ UGC 4499 is inconsistent with $\Lambda \mathrm{CDM}$ since it predicts a too small $c$. Consistency with B99's $\Lambda$ CDM model requires $\Upsilon_{R} \lesssim 0.7(\mathrm{M} / \mathrm{L})_{\odot}$. Since this is not unrealistic, we still consider UGC 4499 to be consistent with CDM.

UGC 5414: The observed rotation velocities imply that $\Upsilon_{R} \lesssim 1.0(\mathrm{M} / \mathrm{L}) \odot$. Furthermore, the data favors a constant density core, and is clearly inconsistent with CDM. However, none of the mass-models provides a realistic fit, even for $\alpha=0$ (either $c<1$ or $f_{\text {bar }}<0.01$ ).

UGC 6446: For the models to be realistic requires $\Upsilon_{R} \lesssim 2.5(\mathrm{M} / \mathrm{L})_{\odot}$. For $\Upsilon_{R}=1.0(\mathrm{M} / \mathrm{L})_{\odot}$ the best-fit model has $\alpha \simeq 1.5$. However, this model is unrealistic (i.e., $c=1$ ), but models with $0<\alpha<1.5$ all provide virtually equally good fits. For $\alpha=1.0$ we find $c \simeq 17.4-10.3 \Upsilon_{R}$ and UGC 6446 is thus consistent with CDM.

UGC 7232: The properties of this galaxy closely resemble those of UGC 5414 and UGC 7323. Even for $\Upsilon_{R}=0$ do we not find a best-fit model with $c>1$ and $f_{\text {bar }}>0.01$. We therefore conclude that no meaningful fit can be obtained for this galaxy. Note that the observed rotation curve consists of only five data points.

UGC 7323: As for UGC 5414 and UGC 7232, no meaningful fit can be obtained for this galaxy.

UGC 7399: In order for the models to be realistic requires $\Upsilon_{R} \lesssim 4(\mathrm{M} / \mathrm{L}) \odot$. For $\alpha=1$ we find $c \simeq 23.1-7.0 \Upsilon_{R}$ and UGC 7339 is thus consistent with CDM.

UGC 7524: This galaxy has the best resolved rotation curve of all galaxies analyzed here. Unfortunately, equation (四) does not yield a reasonable fit to the observed HI surface density, and we therefore opted to use the fullresolution data (see $\S 2$ ) to model the unsmeared HI surface density distribution. The data favors $\Upsilon_{R} \lesssim 4(\mathrm{M} / \mathrm{L})_{\odot}$ and low values for $\alpha$. However, for $\alpha=1$ we find $c \simeq 8.5-3.6 \Upsilon_{R}$, consistent with CDM.

UGC 7559: As is evident from the fact that $\chi_{\text {red }}^{2}>$ 18 , none of our mass-models is able to yield a reasonable fit to the observed rotation curve. However, this is not too surprising since for $r \gtrsim 0.9 \mathrm{kpc}$ the velocity field is highly asymmetric (see S99), which is not properly reflected by the errorbars. As for UGC 5414, UGC 7232 and UGC 7323 no meaningful fit can be obtained.

UGC 7577: No results are plotted for this galaxy, since no model-fit was found to converge. We thus classify this galaxy as UGC 5414, UGC 7232, UGC 7323, and UGC 7603, in that no meaningful fit can be obtained. S99 suggested that UGC 7577, which is at a projected distance of only $37 \mathrm{kpc}$ to NGC 4449, may be a dwarf galaxy that was formed by tidal interactions in the HI streamers around NGC 4449 (Hunter et al. 1998). Such tidal dwarf galaxies are expected to have little or no dark matter (Barnes \& Hernquist 1992).

UGC 7603: Similar to UGC 7524, no reasonable fit to the observed HI surface density can be obtained with equation (11) and we use the full-resolution data to model the unsmeared HI surface density distribution. As for the mass models: no meaningful fit can be obtained.

UGC 8490: The rotation curve is well-resolved and prefers models with a low mass-to-light ratio. For $\alpha=1$ we find $c \simeq 24-10 \Upsilon_{R}$, and this galaxy is thus consistent with CDM.

UGC 9211: Models with $\Upsilon_{R}=1.0(\mathrm{M} / \mathrm{L}) \odot$ provide reasonably good fits to the data, virtually independent of $\alpha$. For $\alpha=1.0$ we find $c \simeq 18.3-3.6 \Upsilon_{R}$, and we thus conclude that this galaxy is consistent with CDM.

UGC 11707: This galaxy reveals a very large amount of freedom in its model parameters. This is partly due to the relatively large errorbars for the inner data points, and which is a reflection of the asymmetry between the receding and approaching rotation velocities at $r \lesssim 7 \mathrm{kpc}$ (see S99). For $\alpha=1.0$ one obtains $c \simeq 13.0-1.8 \Upsilon_{R}$, and we thus conclude that this galaxy is consistent with CDM.

UGC 11861: This is one of the few galaxies for which $\chi_{\text {red }}^{2}$ decreases with increasing $\Upsilon_{R}$ (see also UGC 5424, UGC 8490, and, to a lesser extent, UGC 7524 and UGC 12732). For $\Upsilon_{R} \gtrsim 3(\mathrm{M} / \mathrm{L}) \odot$ the implied baryon fraction becomes unrealistically large. For $\alpha=1$ we find $c \simeq 16-3 \Upsilon_{R}$, and we thus conclude that this galaxy is consistent with CDM.

UGC 12060: The rotation curve of this galaxy is well fitted by the mass models. As for UGC 11707, there is a large amount of freedom in the model parameters. For $\alpha=1$ we obtain $c \simeq 34.6-9.8 \Upsilon_{R}$. This implies that according to our definition UGC 12060 is inconsistent with $\Lambda$ CDM for $\Upsilon_{R}=0$, since the best fit halo concentration is too large (i.e., $\left.c=34.6>c_{\max }=31.9\right)$. However, for more realistic massto-light ratios the best-fit halo concentration is in excellent agreeement with the predictions of the B99 model, as we thus conclude that this galaxy is consistent with CDM.

UGC 12632: The properties of UGC 12632 are similar to those of UGC 731. For $\alpha=1.0$ we find $c \simeq 14.0-1.3 \Upsilon_{R}$, and we thus conclude that this galaxy is consistent with CDM.

UGC 12732: For this galaxy, larger mass-to-light ratios imply smaller values for $\alpha$. For $\Upsilon_{R} \gtrsim 3.5(\mathrm{M} / \mathrm{L}) \odot$ the 
resulting baryon fraction becomes unrealistically small. For $\Upsilon_{R}=1.0(\mathrm{M} / \mathrm{L})_{\odot}$, the best-fitting model has $\alpha=1.5$, but also $c=1$, and is thus unrealistic. For $\alpha=1.0$ we find $c \simeq 10.3-3.3 \Upsilon_{R}$, and we thus conclude that this galaxy is consistent with CDM. 


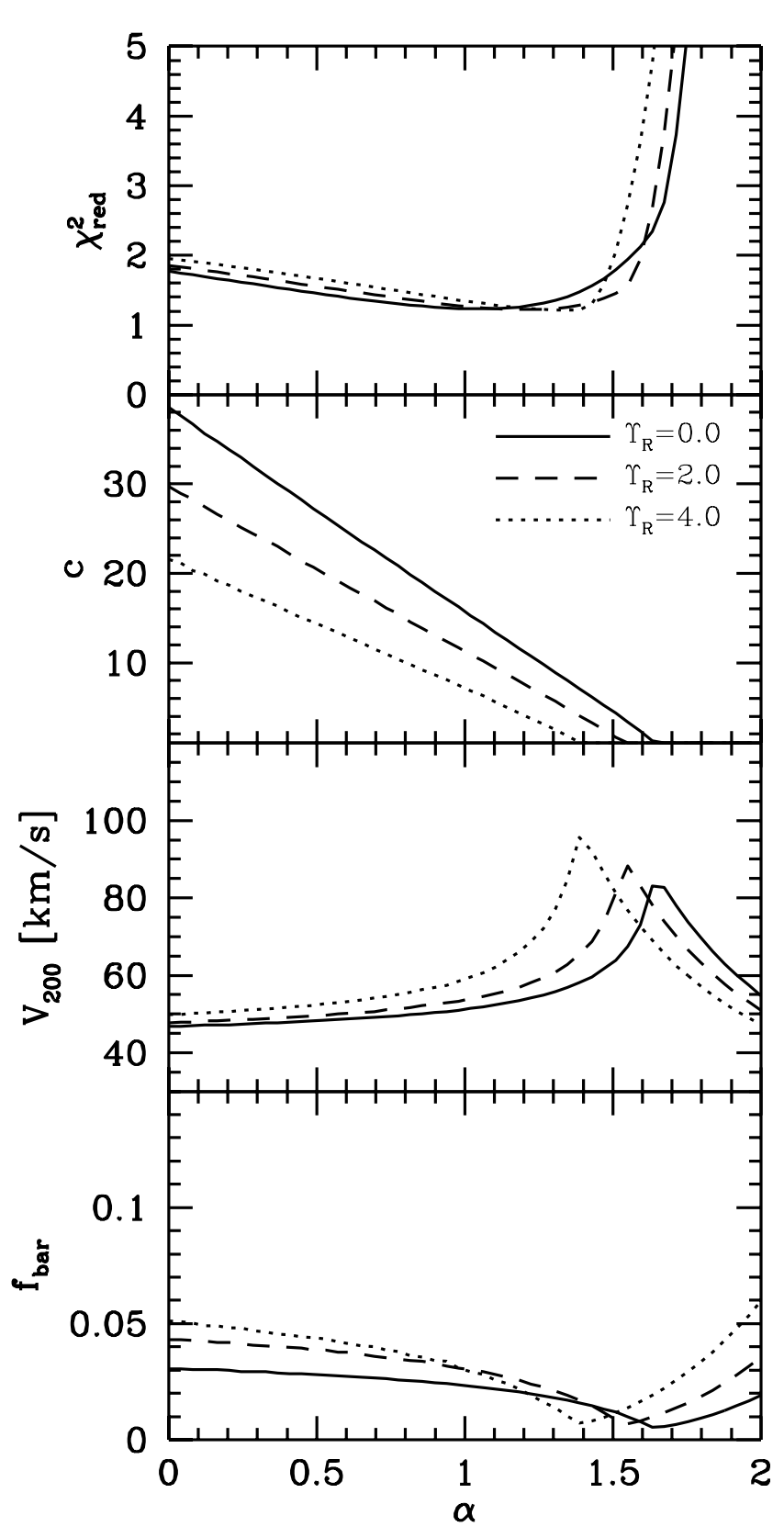

UGC 731
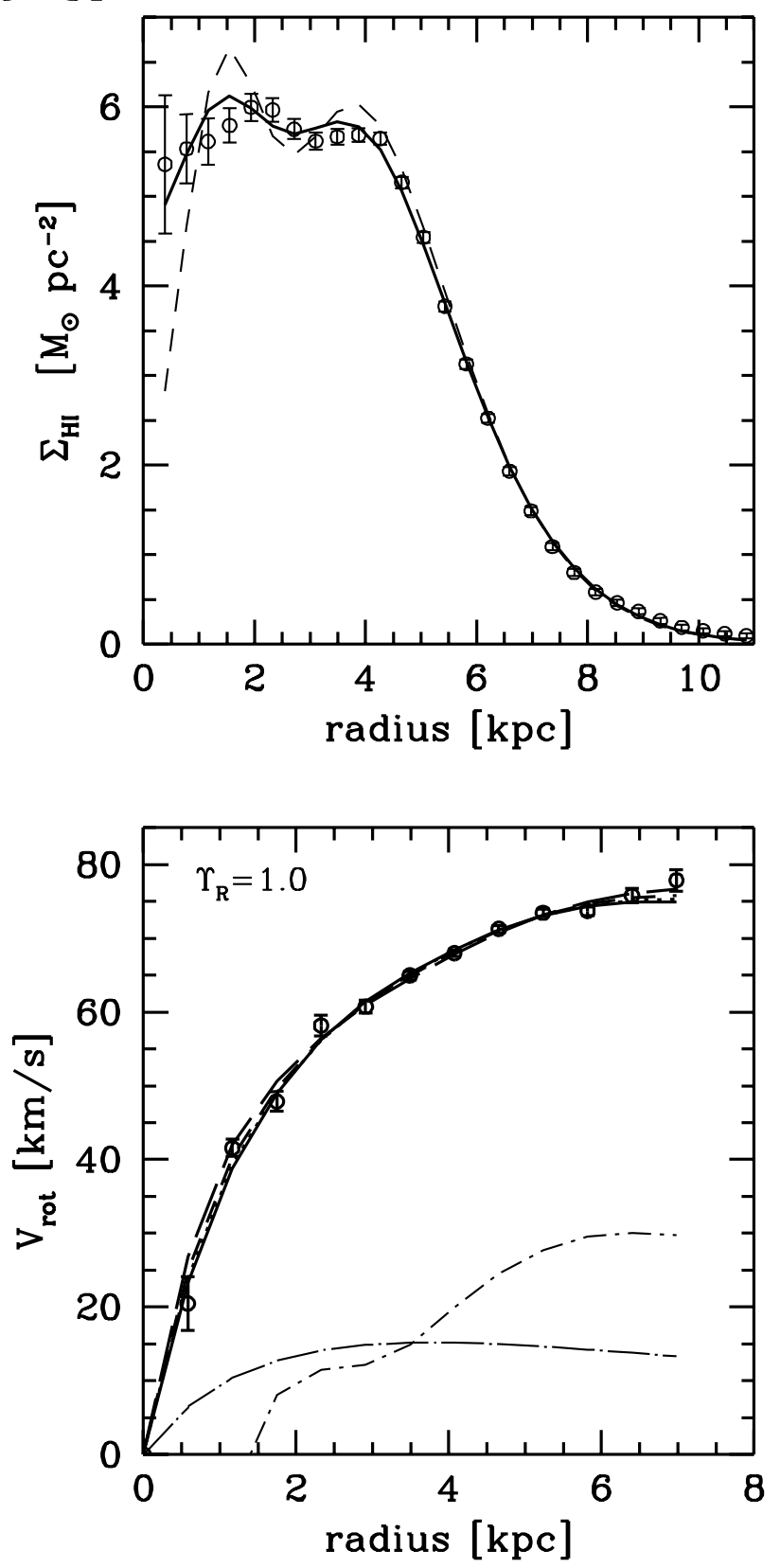

Figure A1. The panel in the upper-right corner plots the observed HI surface density (open circles with errorbars) together with the best-fit model of equation (11) both before (dashed lines) and after (solid lines) beam smearing. In the cases of UGC 7524 and UGC 7603 , were no acceptable model fit can be obtained, the dashed lines indicate the observed HI surface density at the full resolution of the observations (see $\S$ ), which we use as a model for the unsmeared surface density of the HI. The four panels on the left show, from top to bottom, $\chi_{\text {red }}^{2}, c, V_{200}$, and $f_{\text {bar }}$ of the best-fit models, as functions of $\alpha$ and for three different mass-to-light ratios (as indicated in the second panel). The lower-right corner panel, finally, plots the observed rotation curve (open circles with errorbars) together with four best-fit models with $\Upsilon_{R}=1.0(\mathrm{M} / \mathrm{L}) \odot: \alpha=0$ (solid lines), $\alpha=0.5$ (dotted lines), $\alpha=1.0$ (short-dash lines) and $\alpha=1.5$ (long-dash lines). These four models only differ in their dark matter properties; they have the same gaseous and stellar disks, the contributions of which are also indicated by (dot - short-dash) and (dot - long-dash) lines, respectively. These plots are useful for assessing the typical quality of the model fits and the cusp-core degeneracy discussed in the text. 
UGC 3371
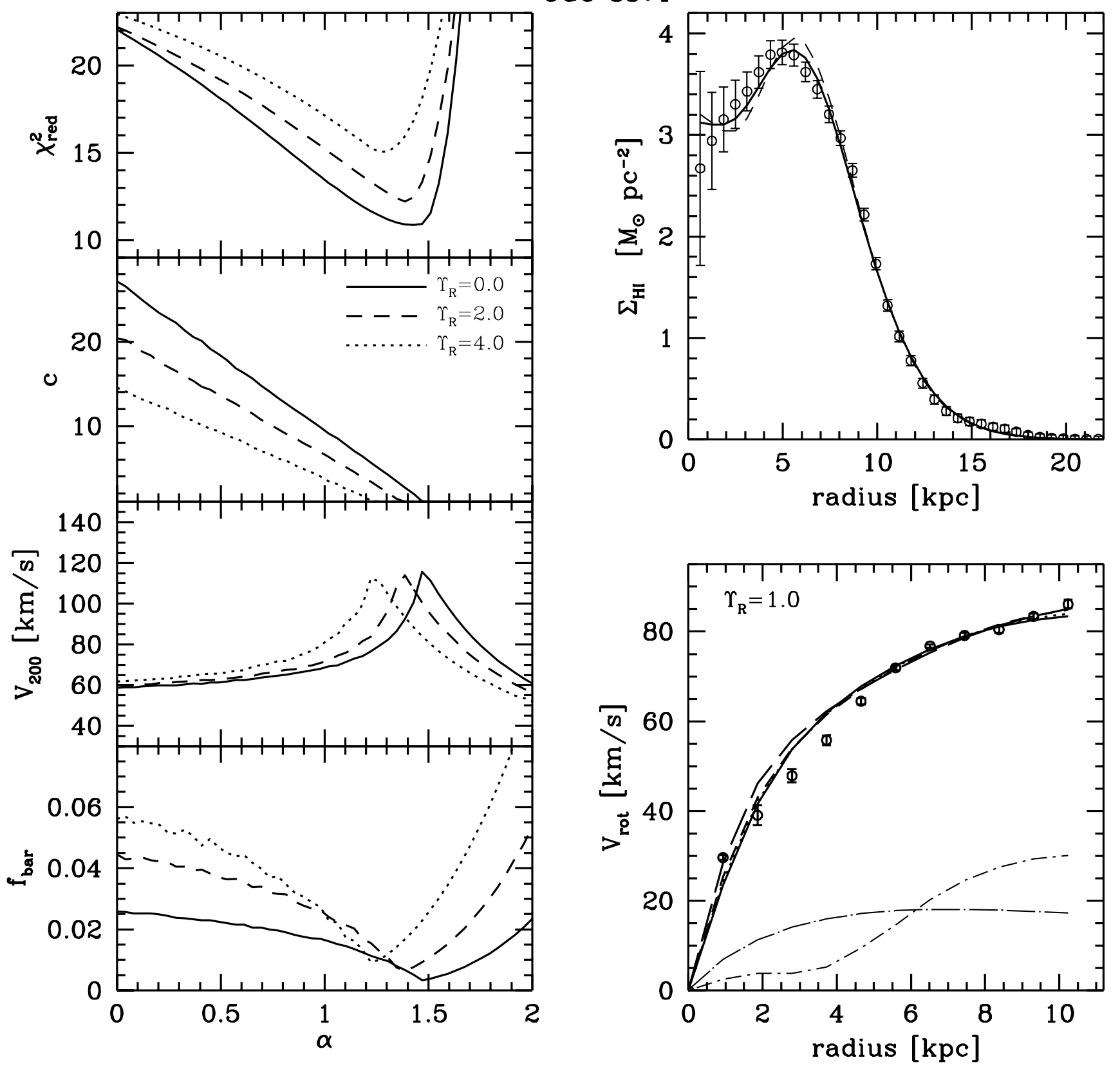

Figure A2. Same as Figure A1 but for UGC 3371 


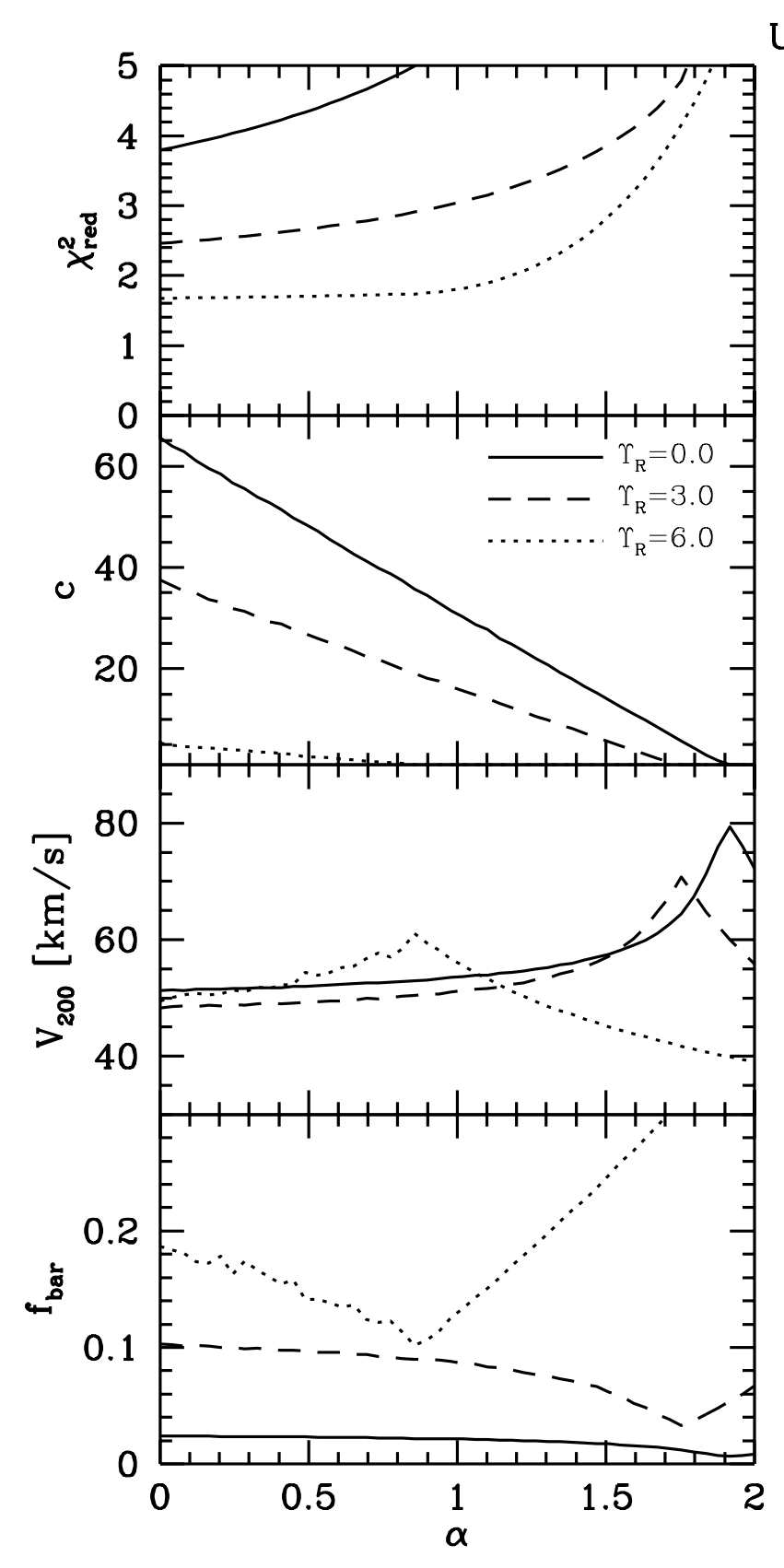

UGC 4325
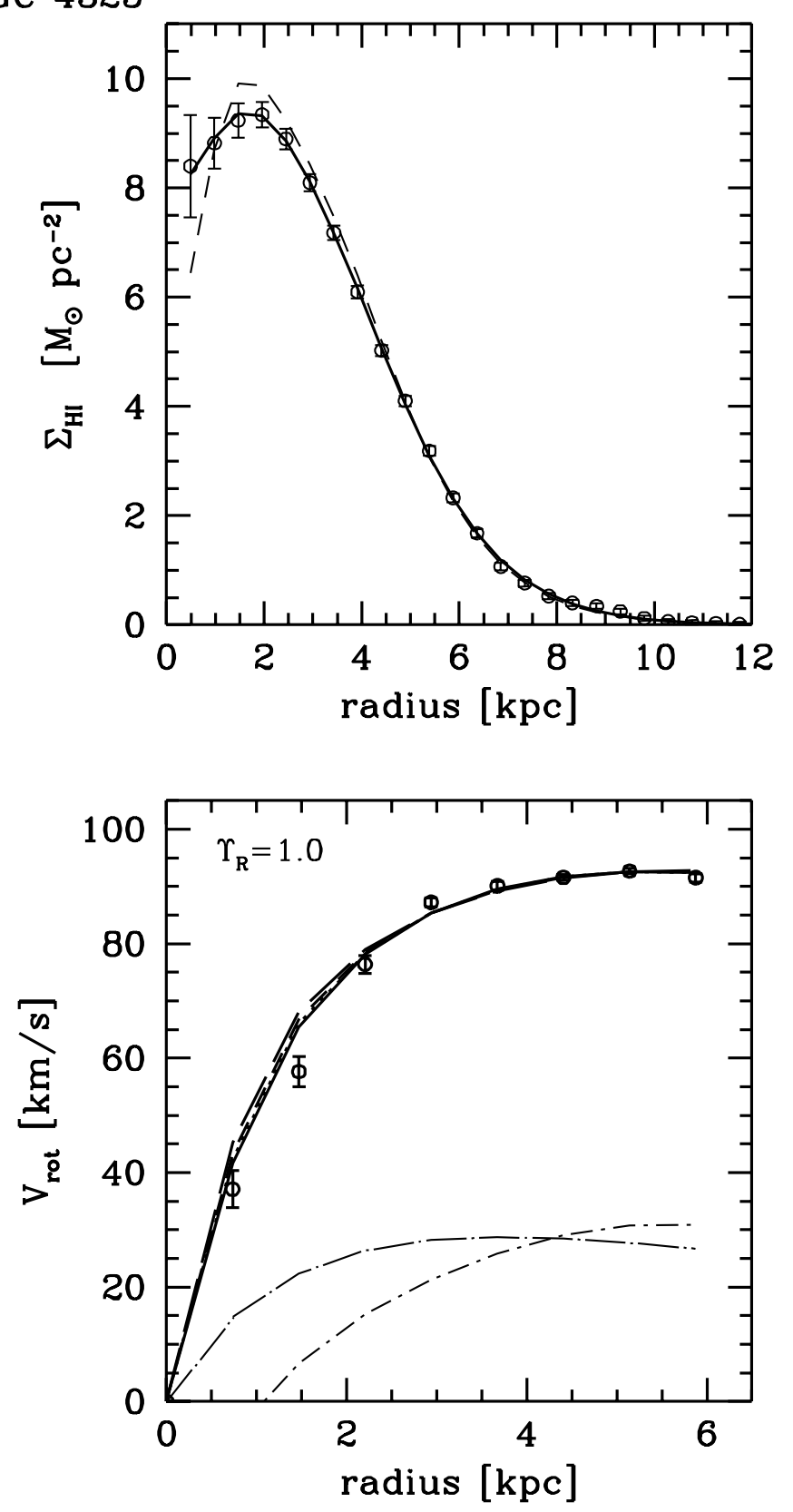

Figure A3. Same as Figure A1 but for UGC 4325 
UGC 4499
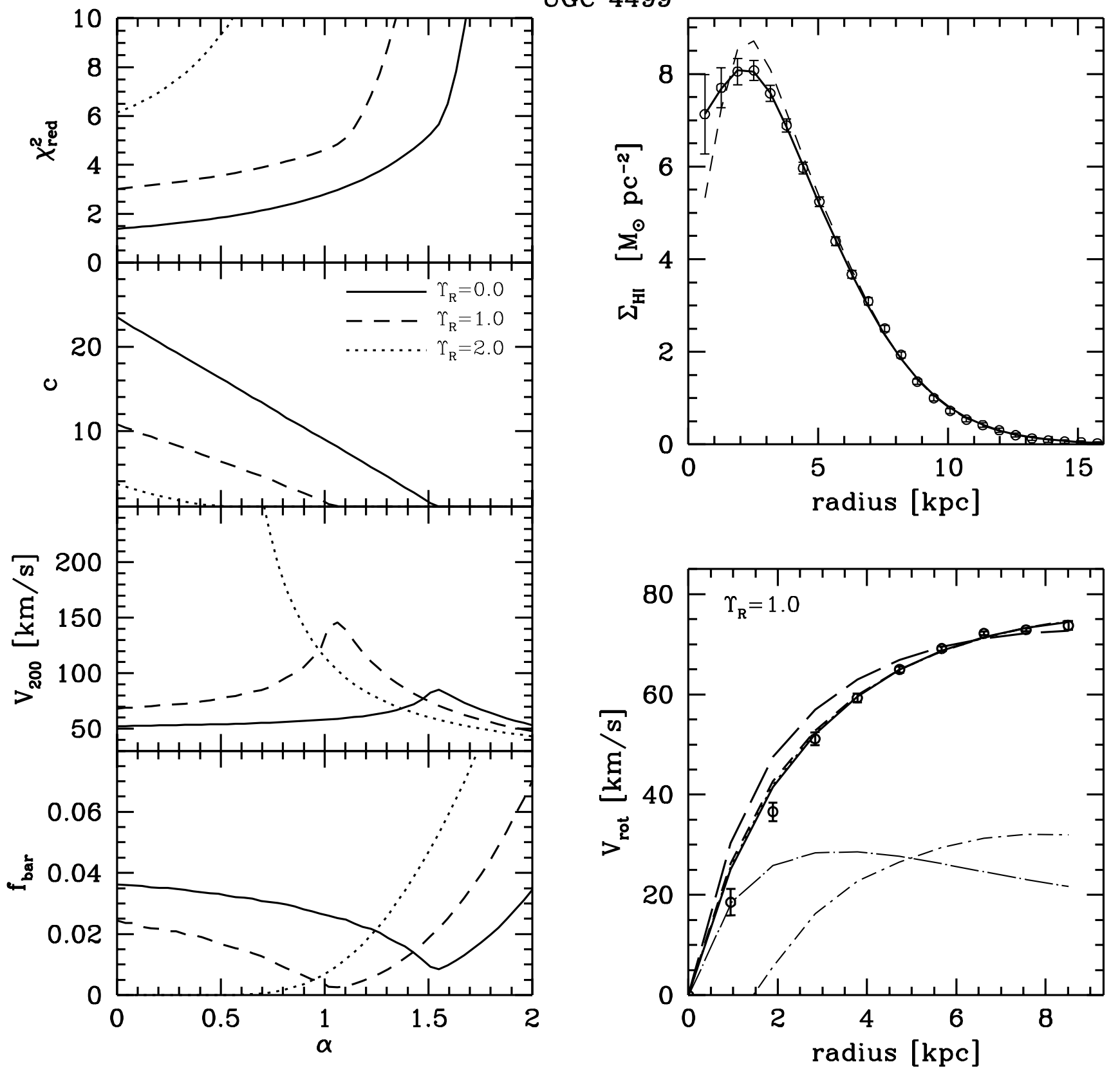

Figure A4. Same as Figure A1 but for UGC 4499 


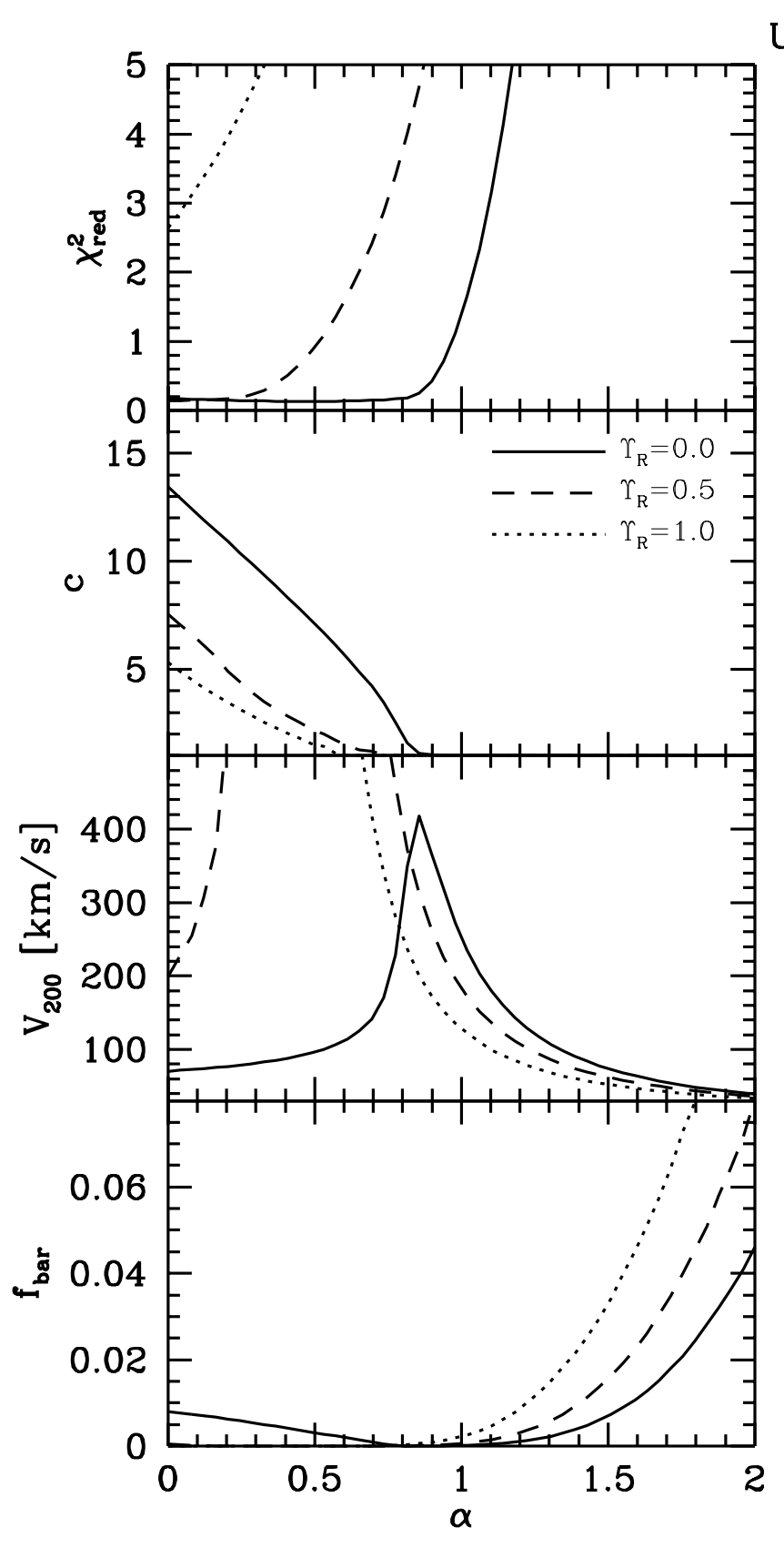

UGC 5414
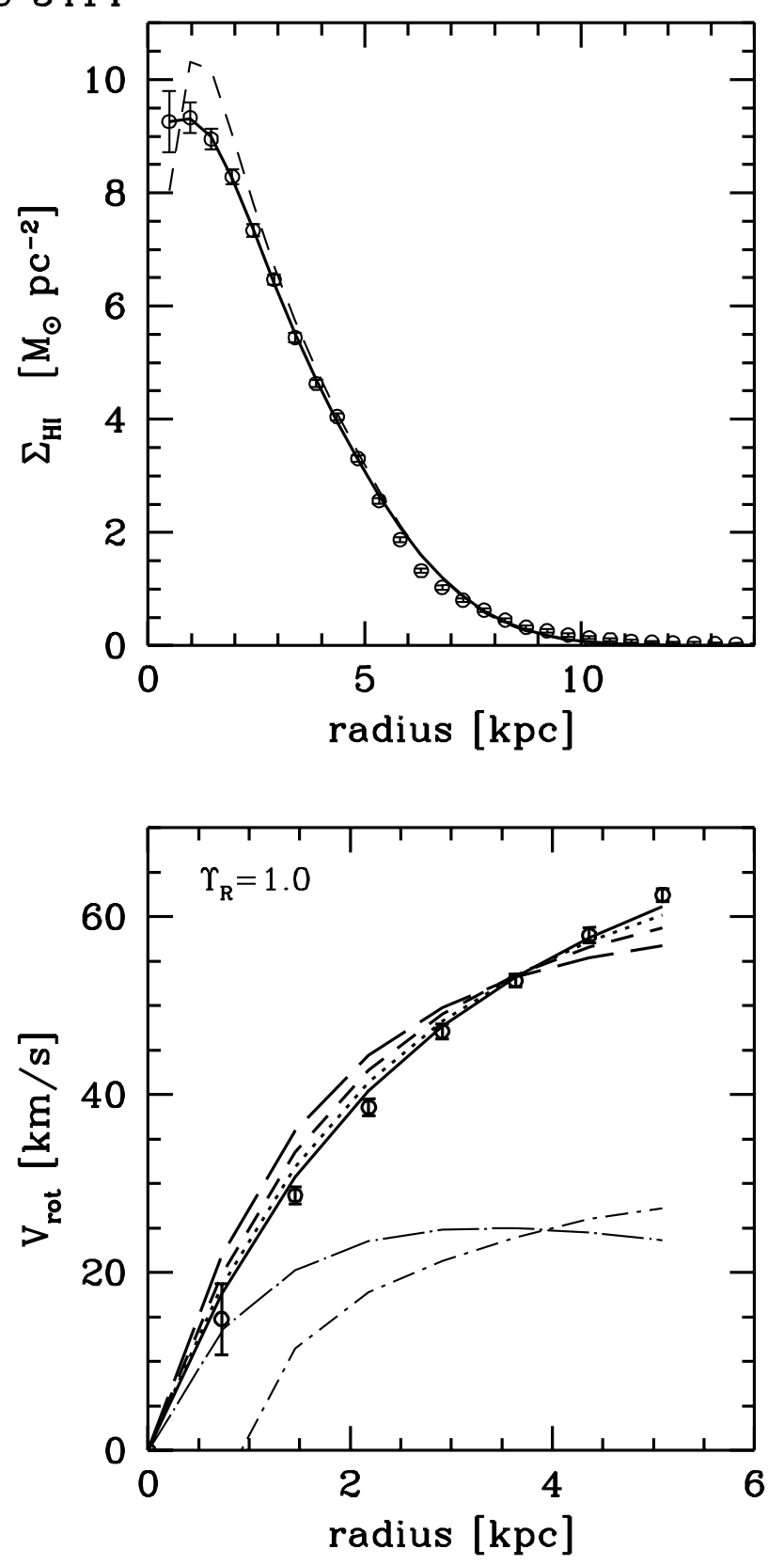

Figure A5. Same as Figure A1 but for UGC 5414 


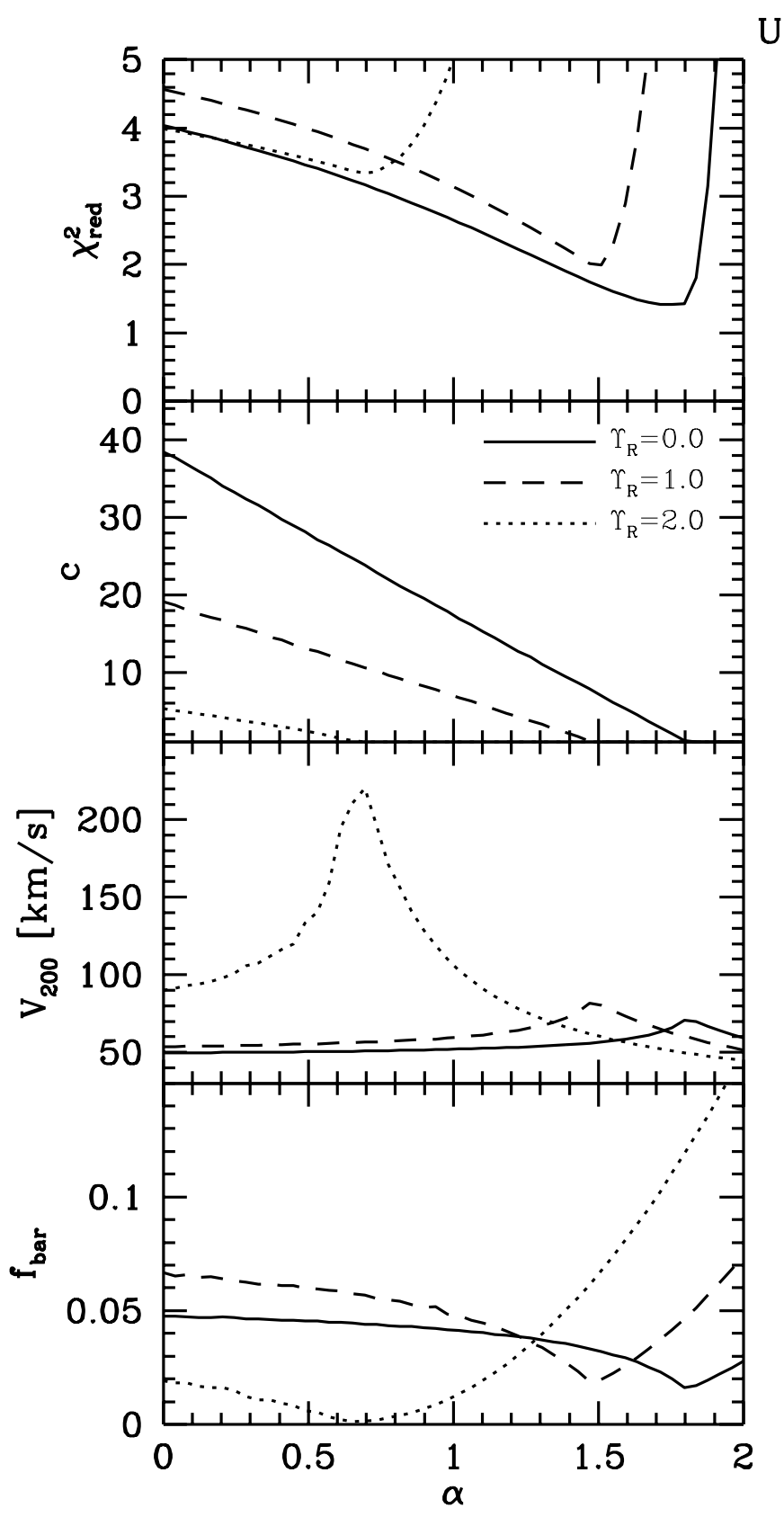

UGC 6446
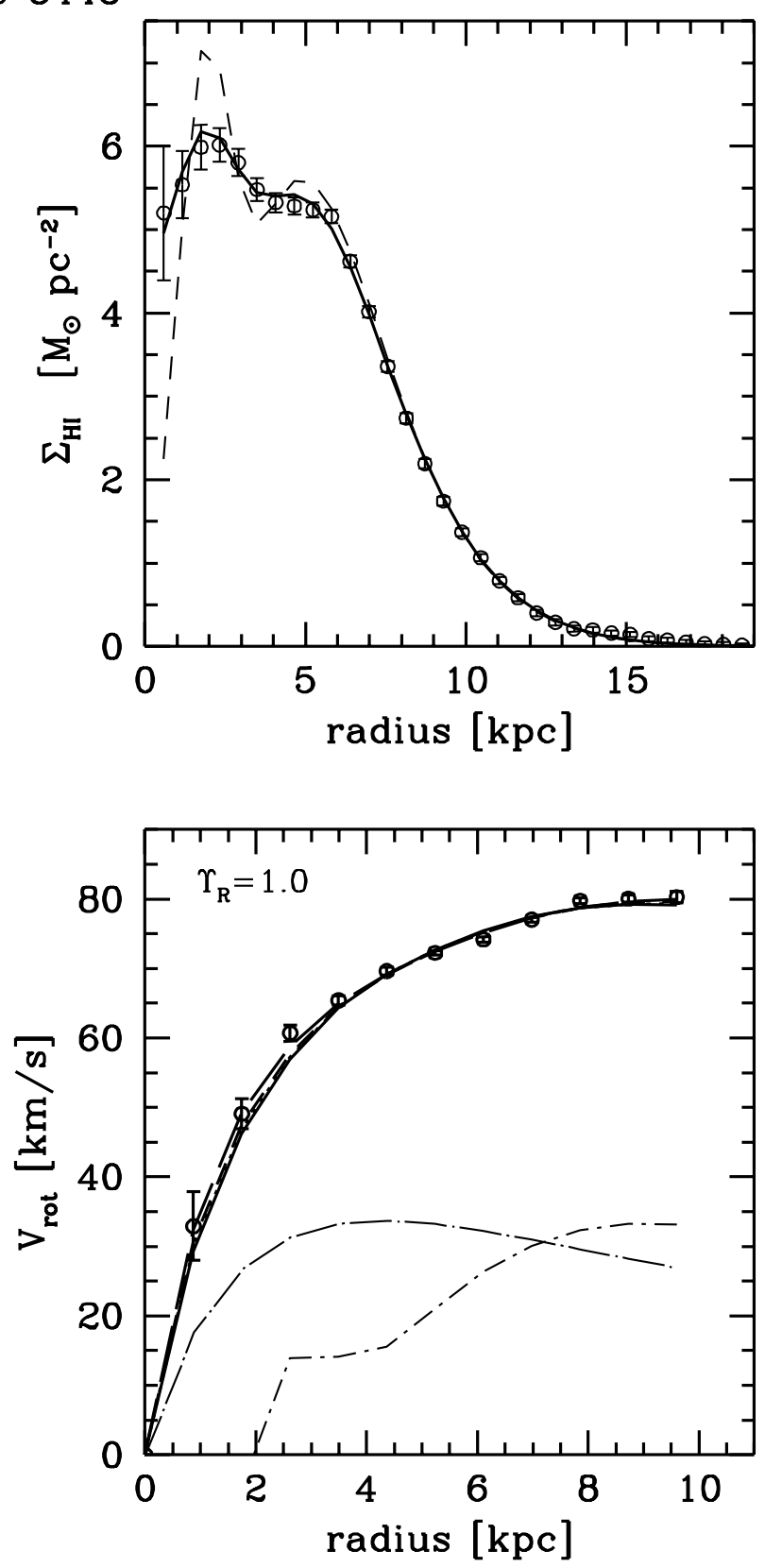

Figure A6. Same as Figure A1 but for UGC 6446 


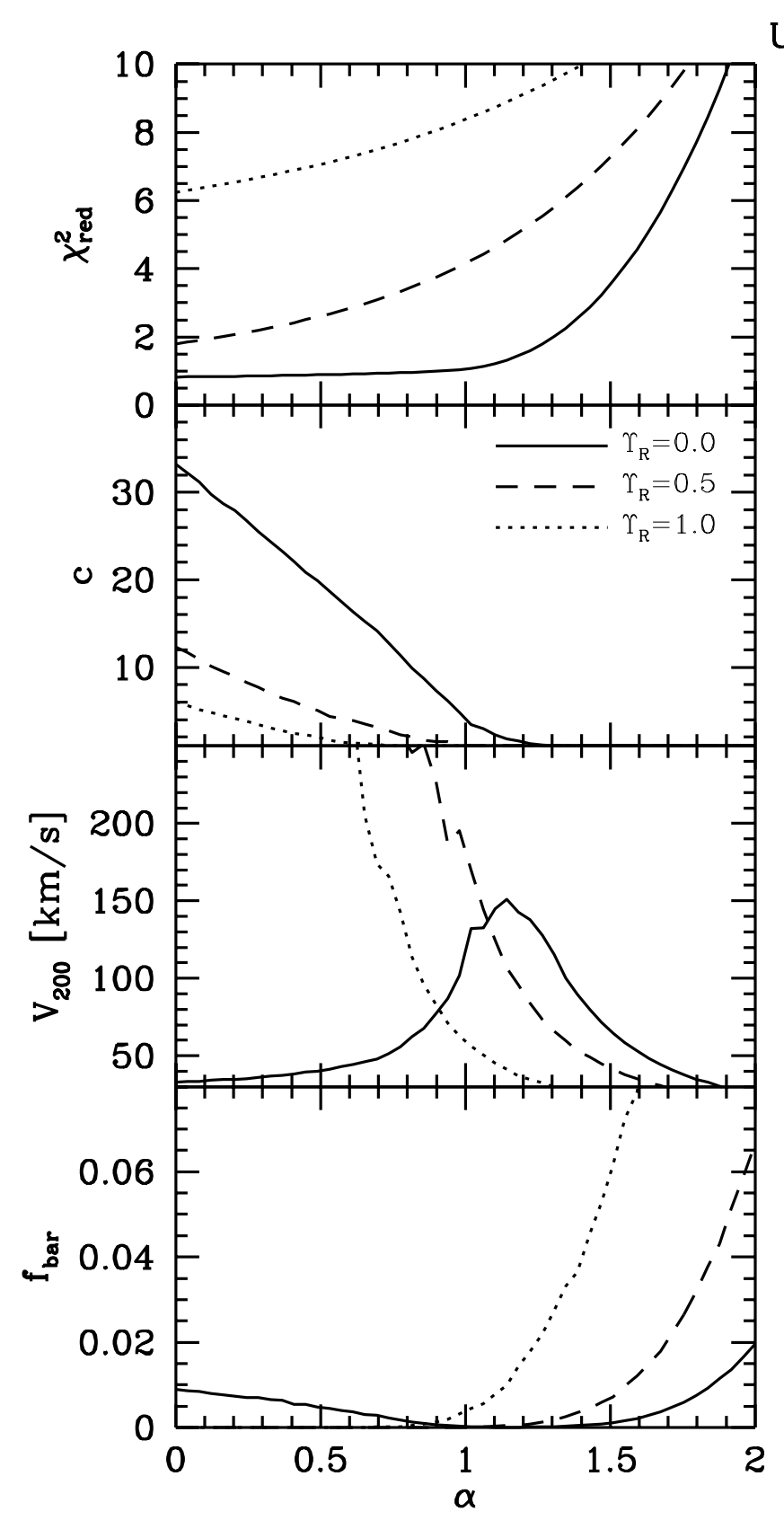

UGC 7232
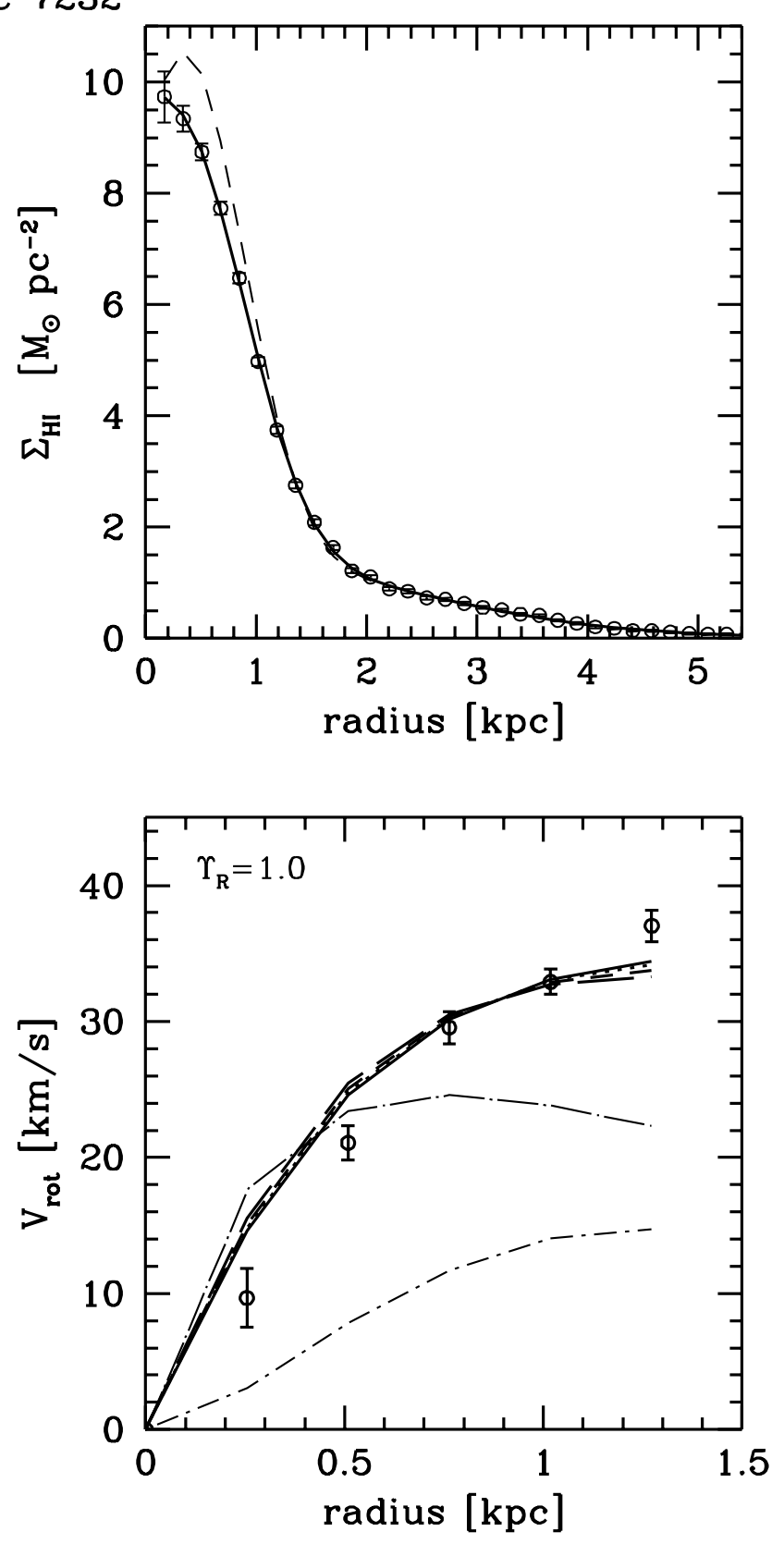

Figure A7. Same as Figure A1 but for UGC 7232 


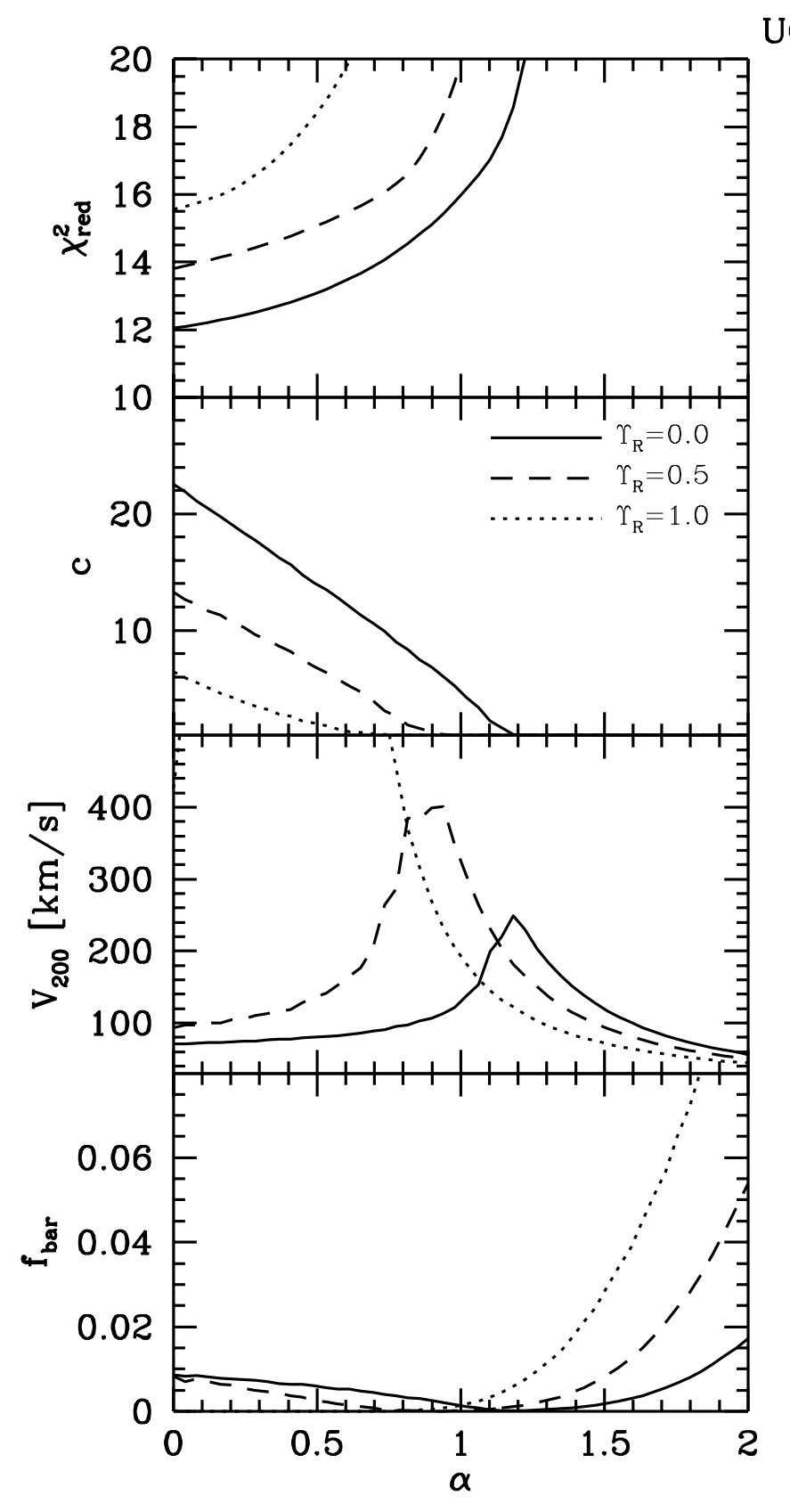

UGC 7323
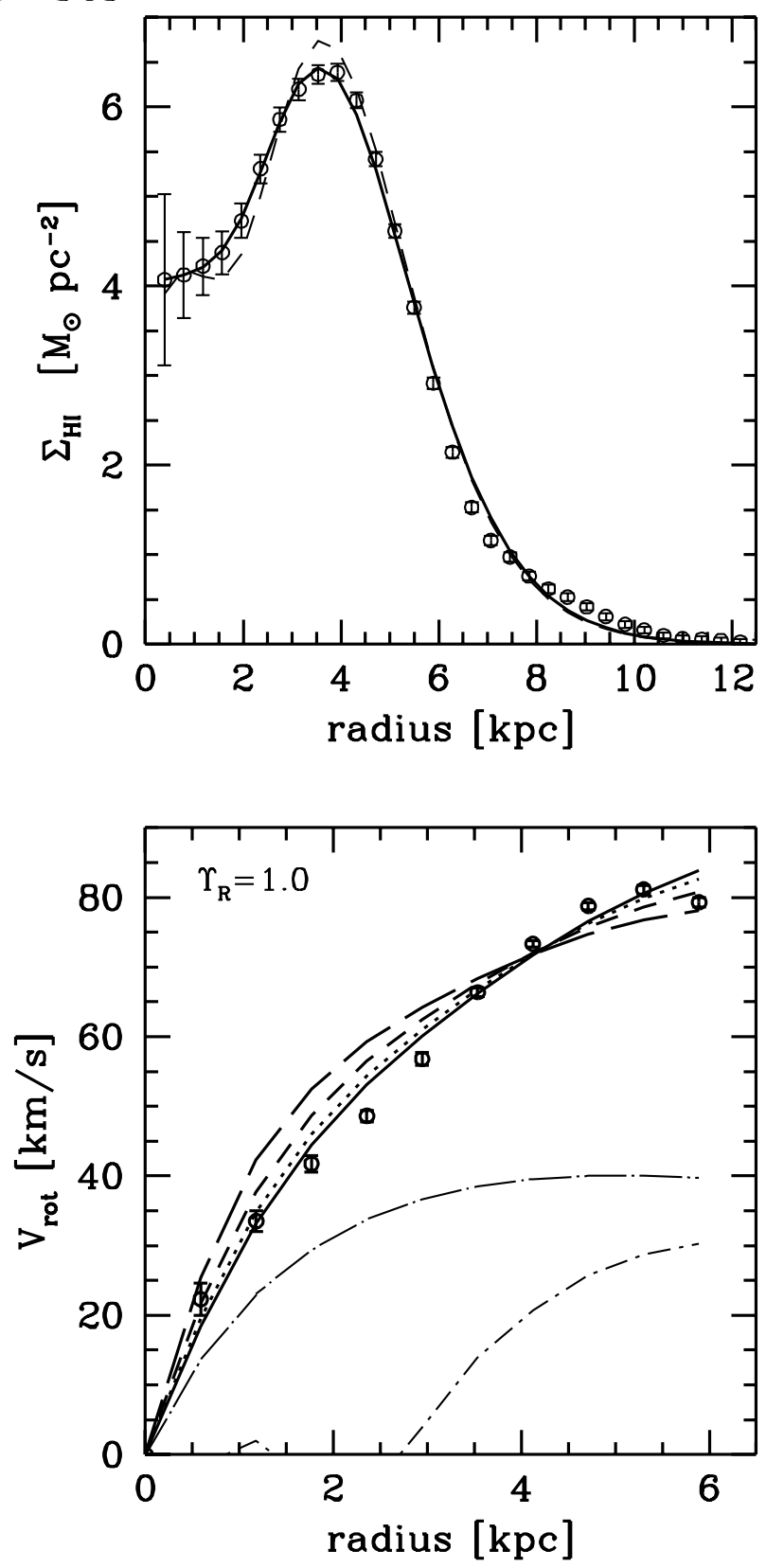

Figure A8. Same as Figure A1 but for UGC 7323 
UGC 7399
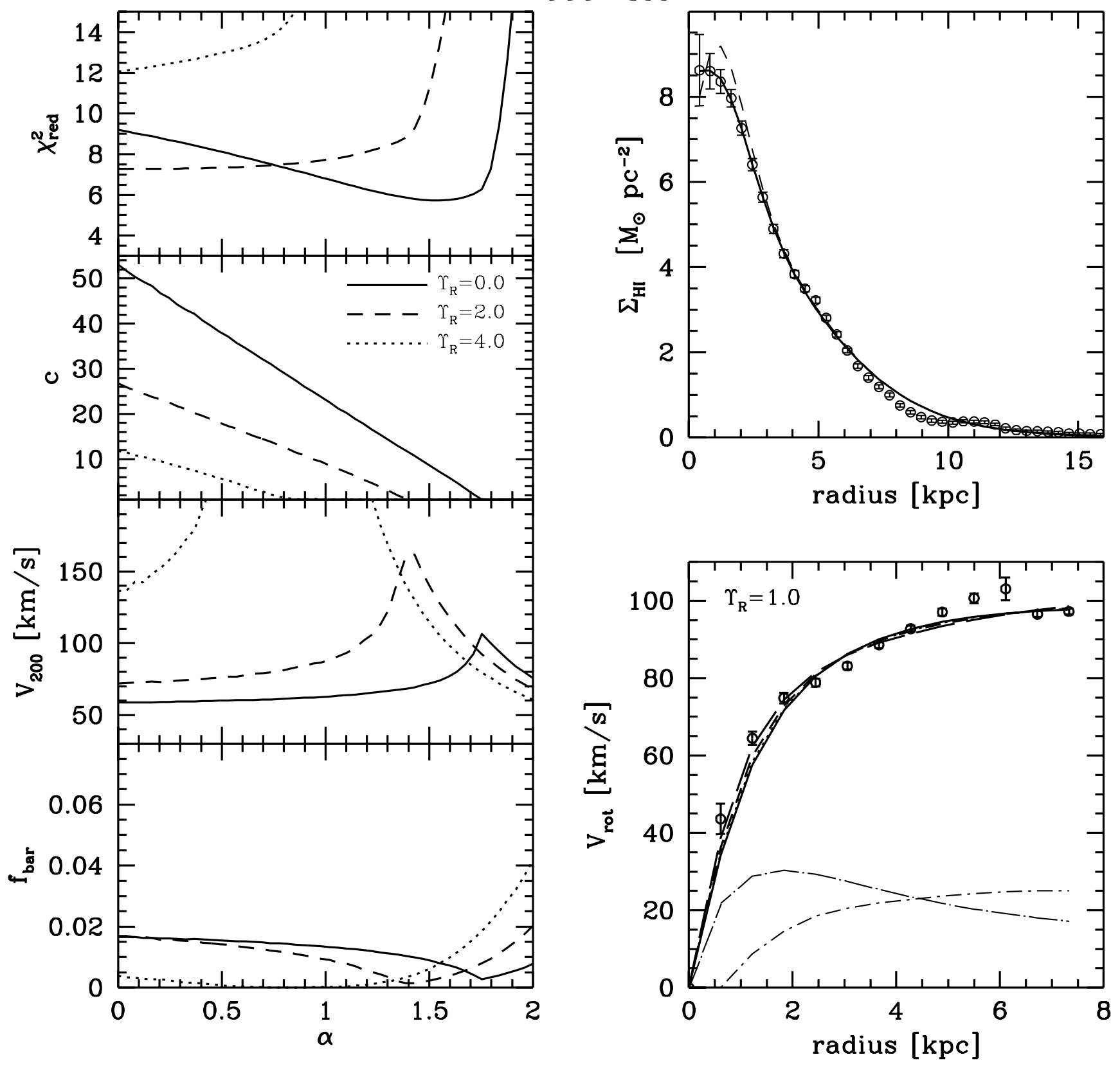

Figure A9. Same as Figure A1 but for UGC 7399 


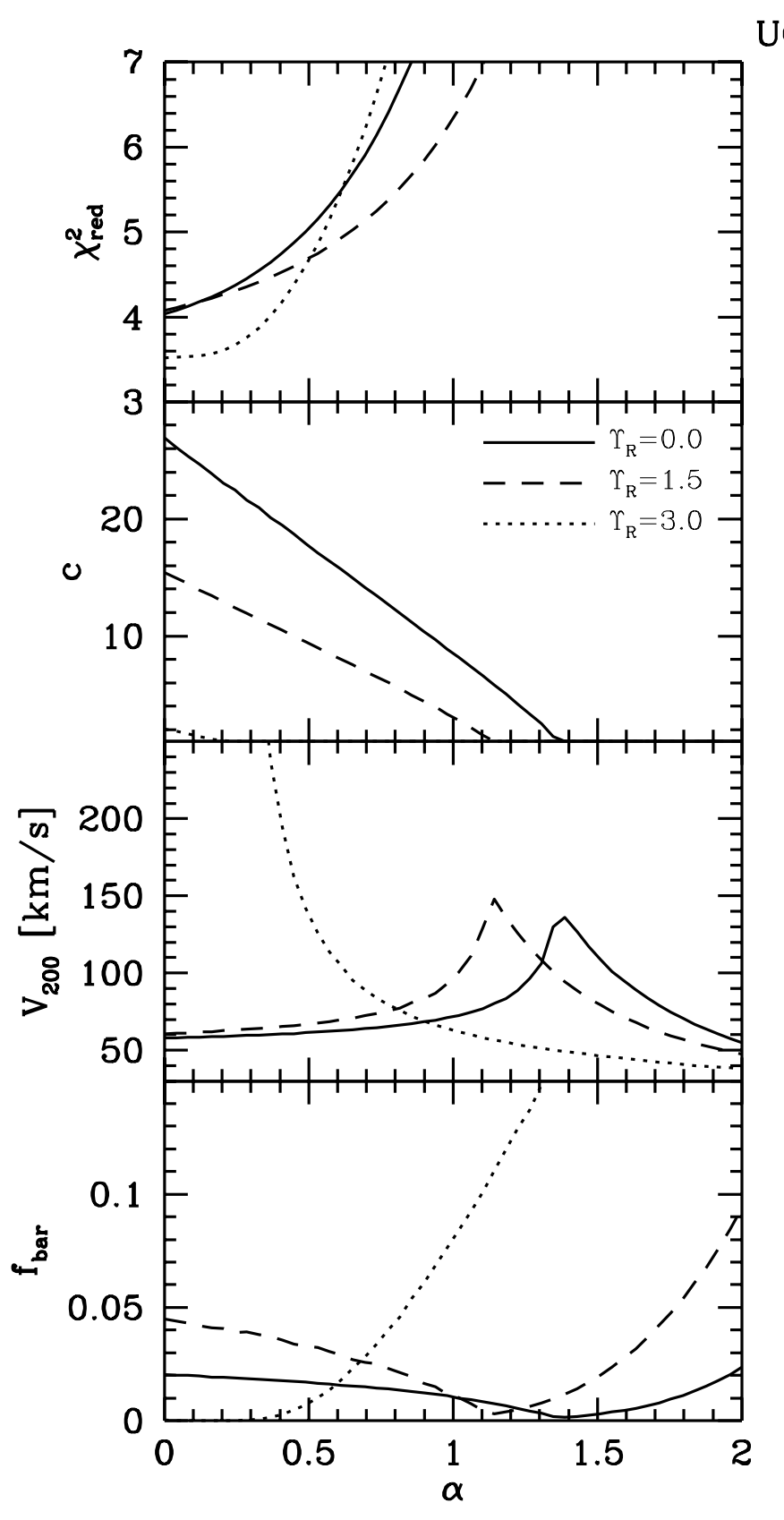

UGC 7524
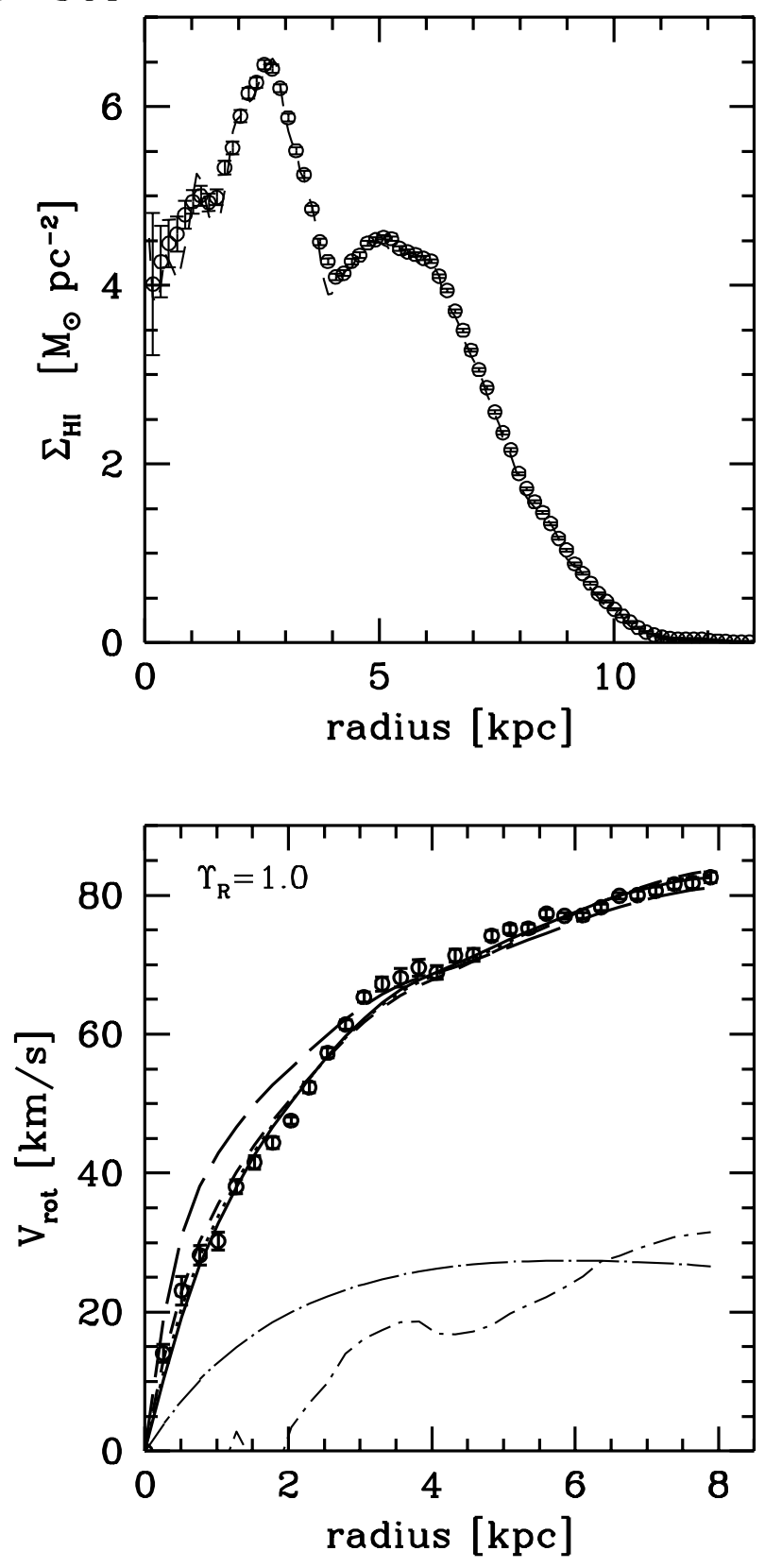

Figure A10. Same as Figure A1 but for UGC 7524 


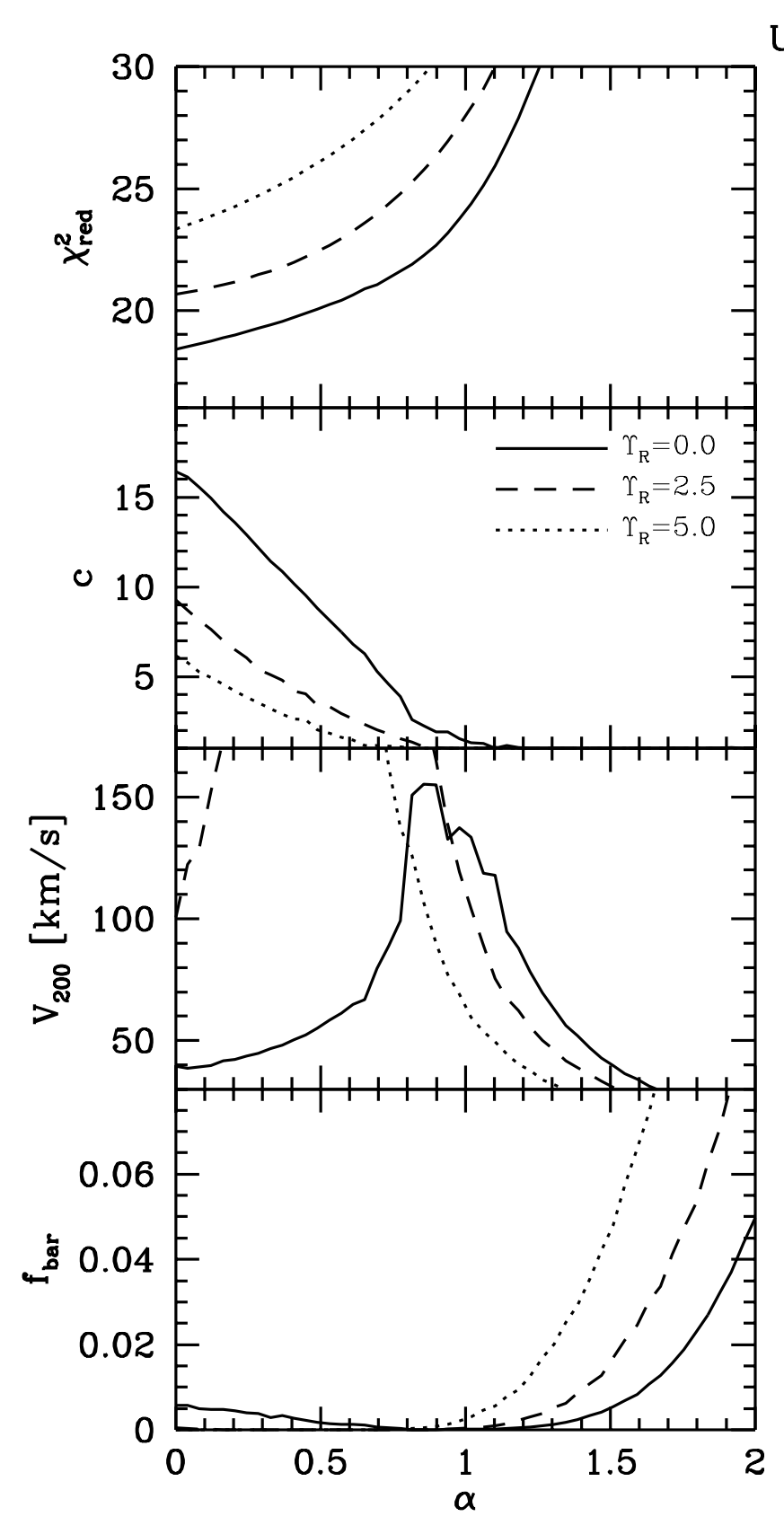

UGC 7559
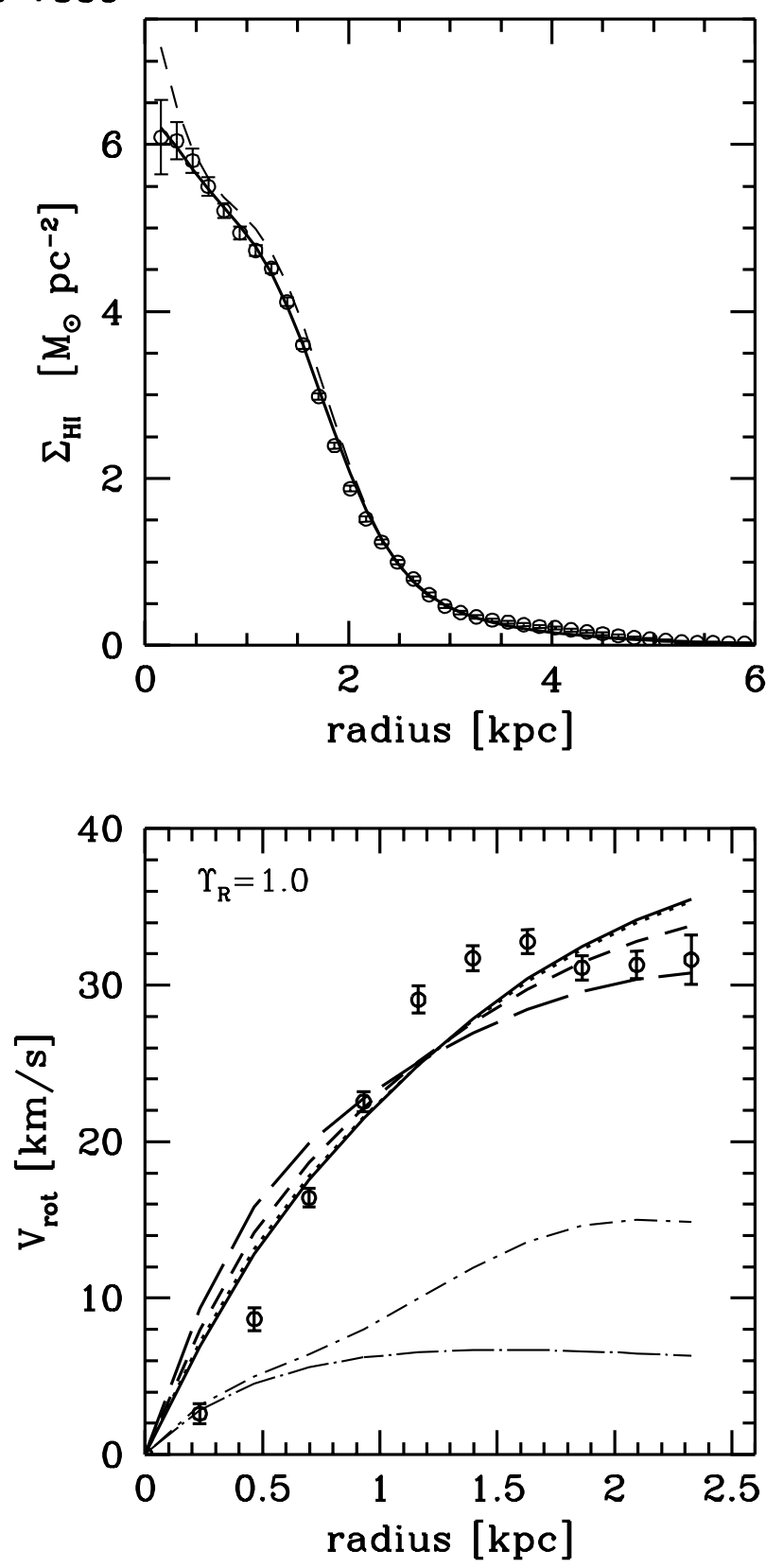

Figure A11. Same as Figure A1 but for UGC 7559 
UGC 7603
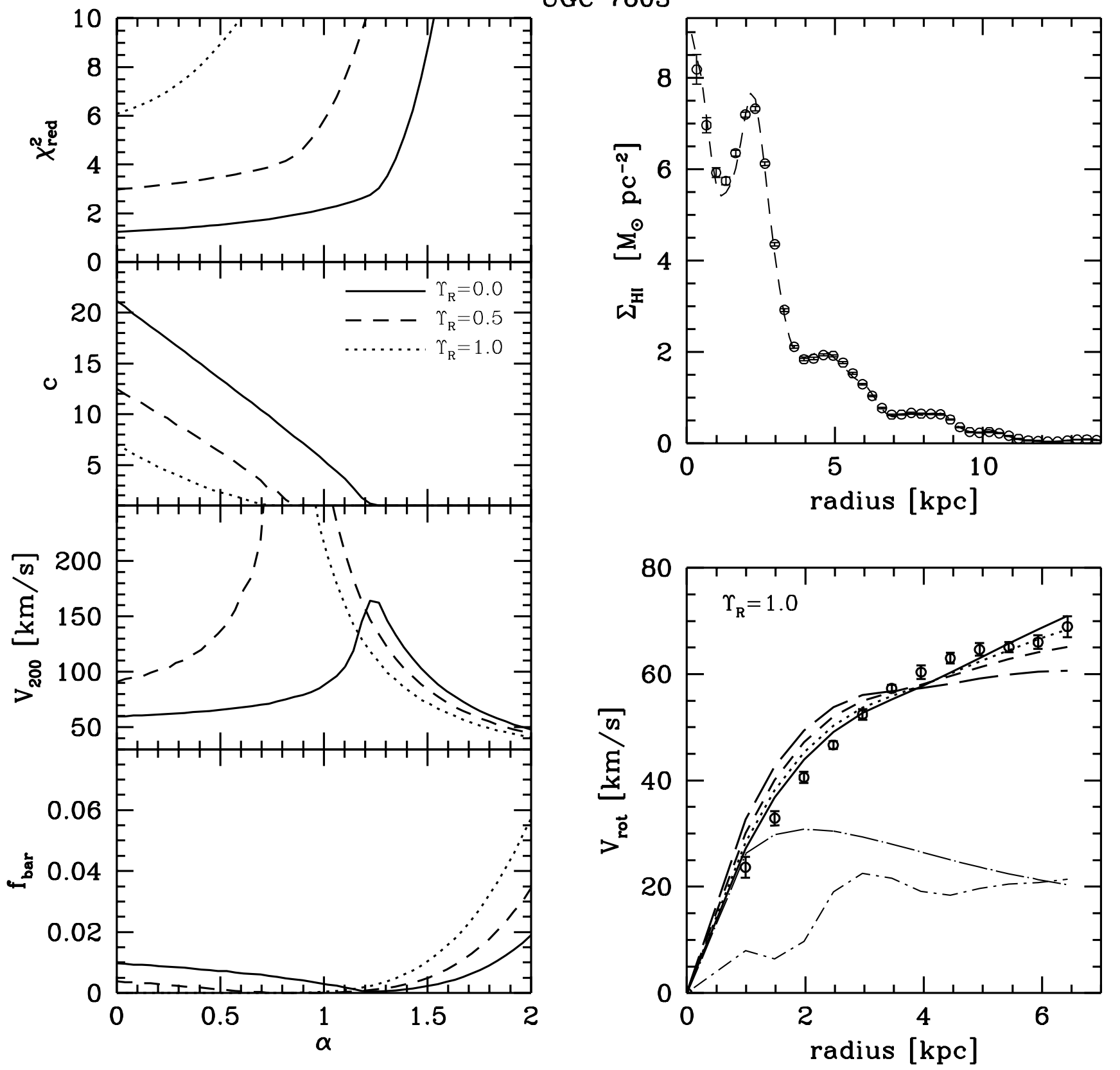

Figure A12. Same as Figure A1 but for UGC 7603 


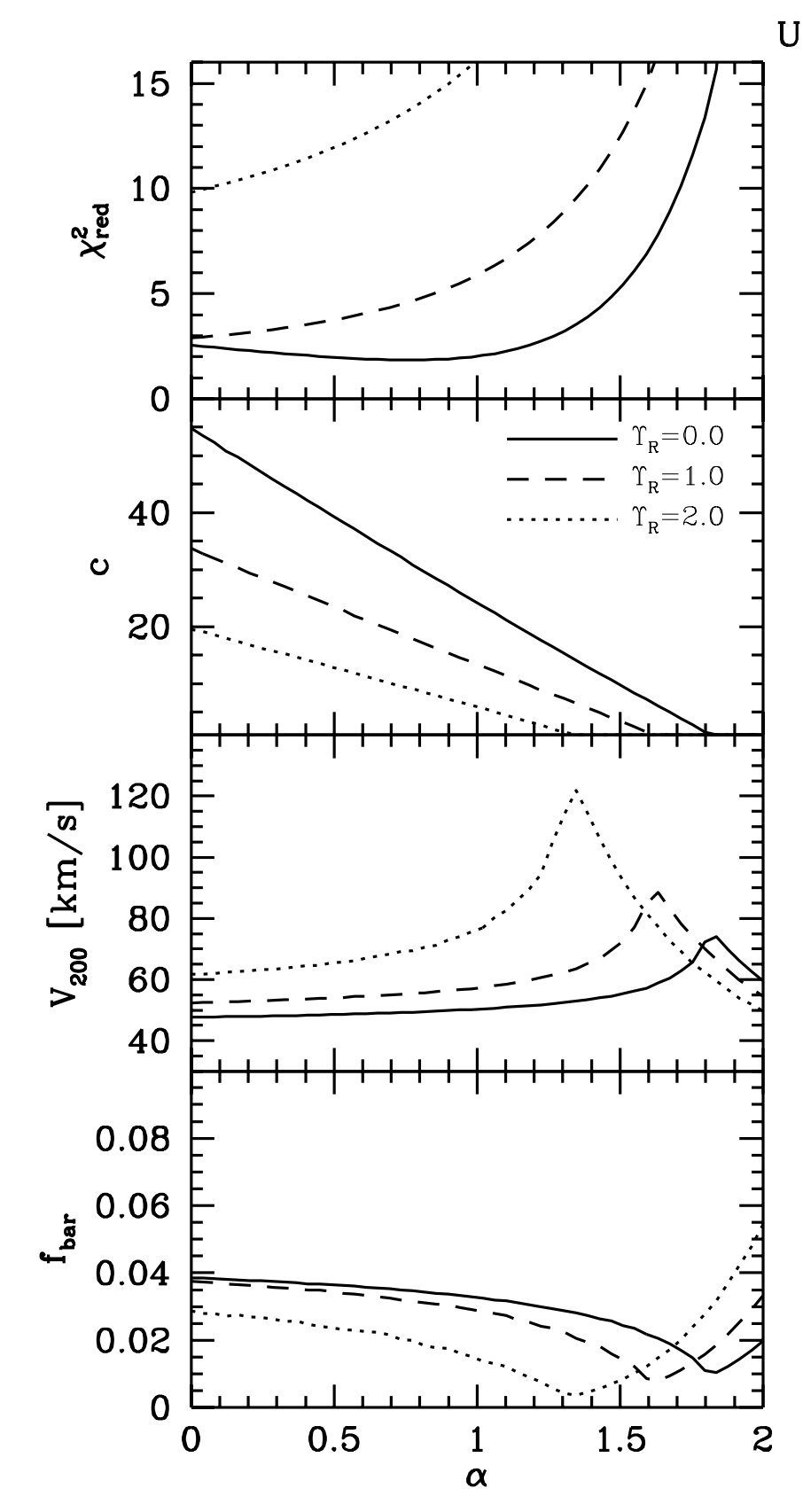

UGC 8490
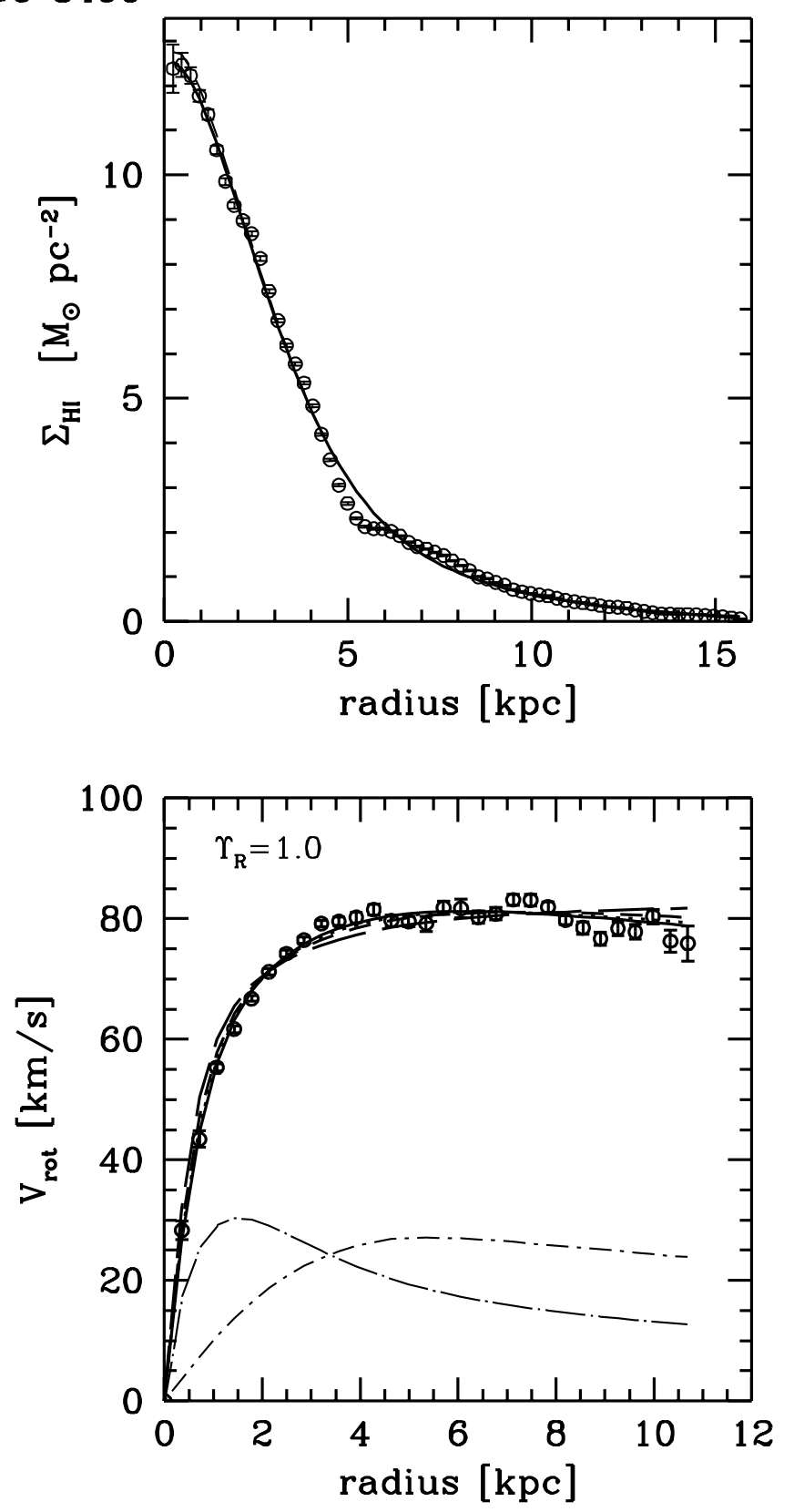

Figure A13. Same as Figure A1 but for UGC 8490 
UGC 9211
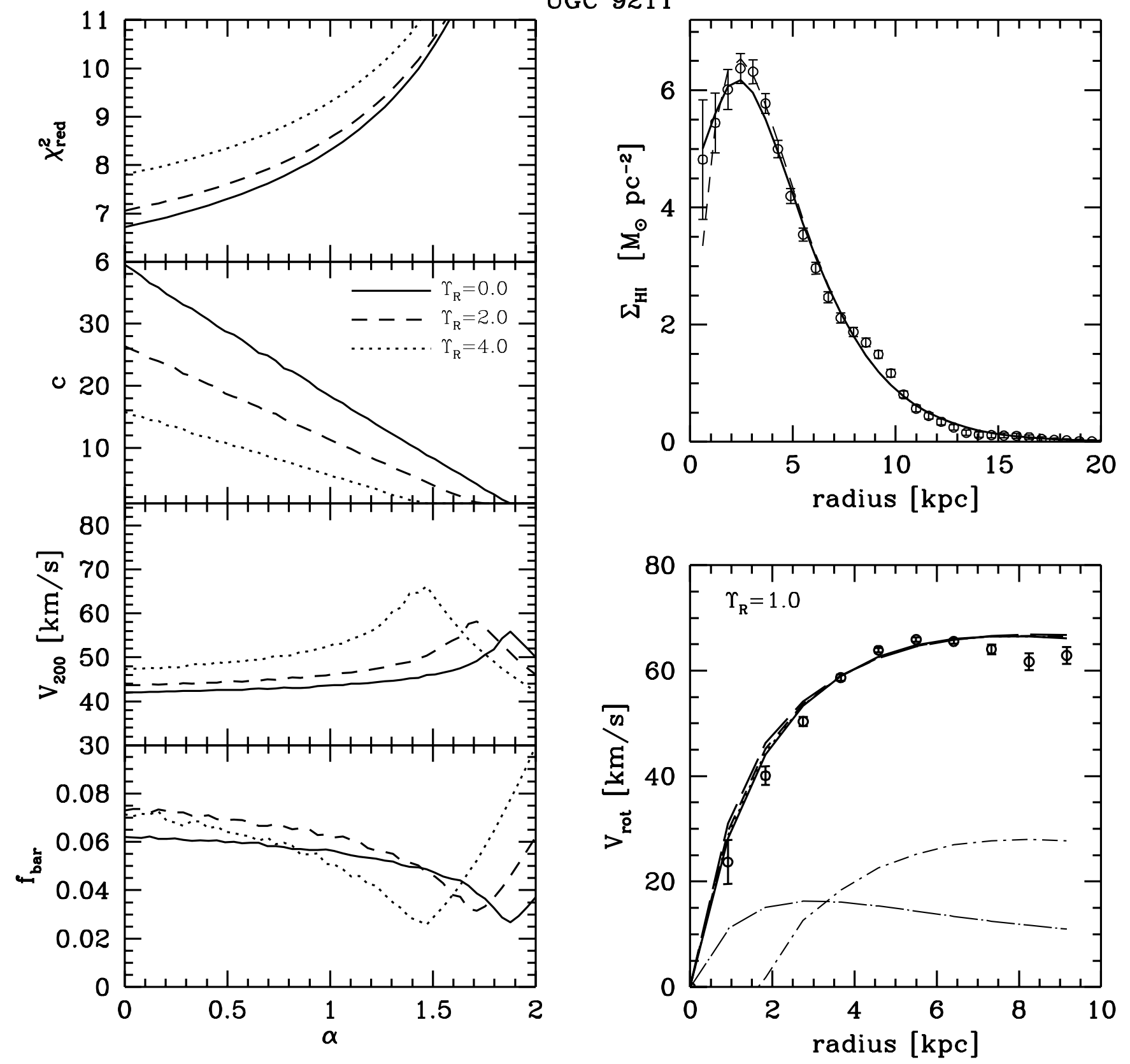

Figure A14. Same as Figure A1 but for UGC 9211 


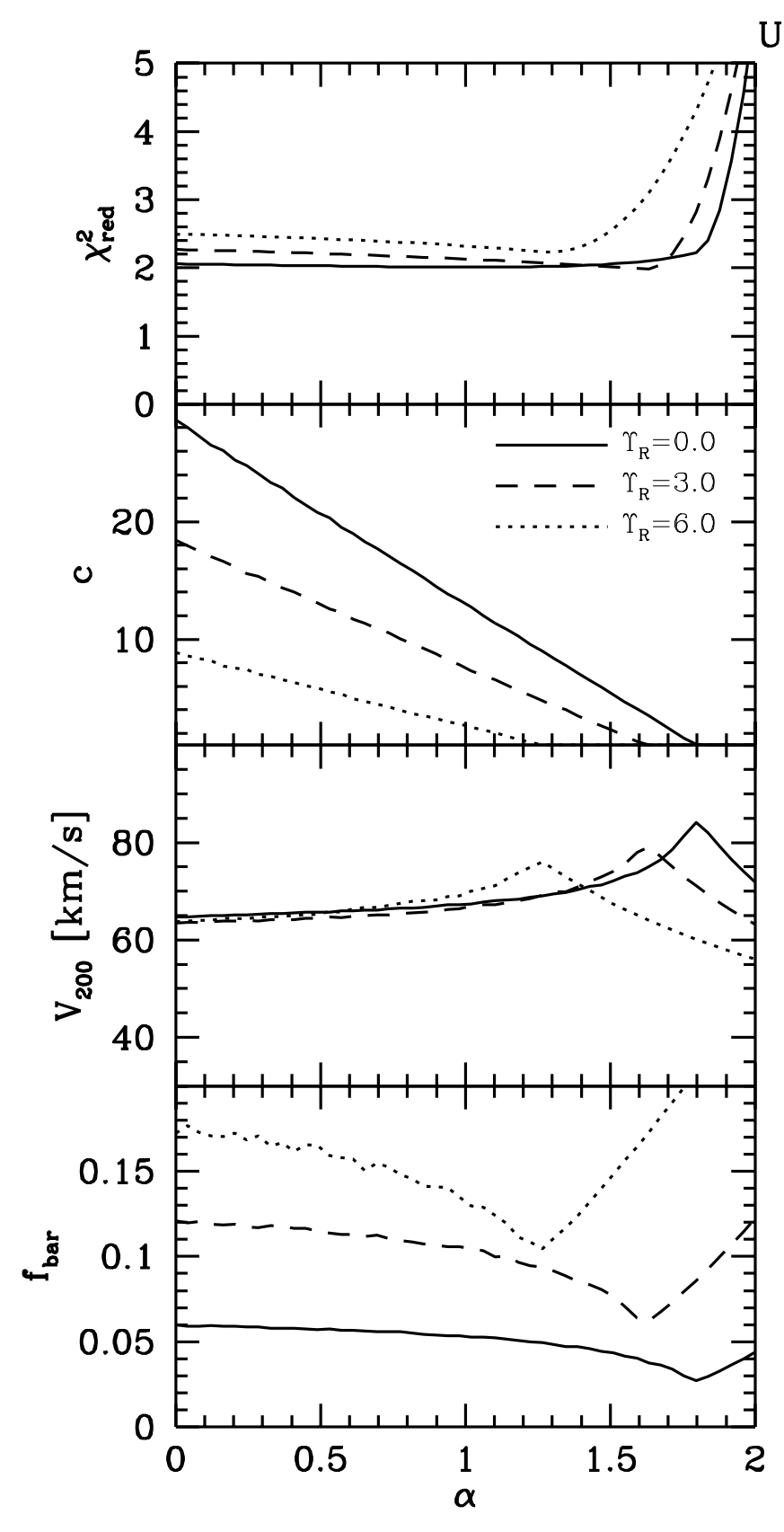

UGC 11707
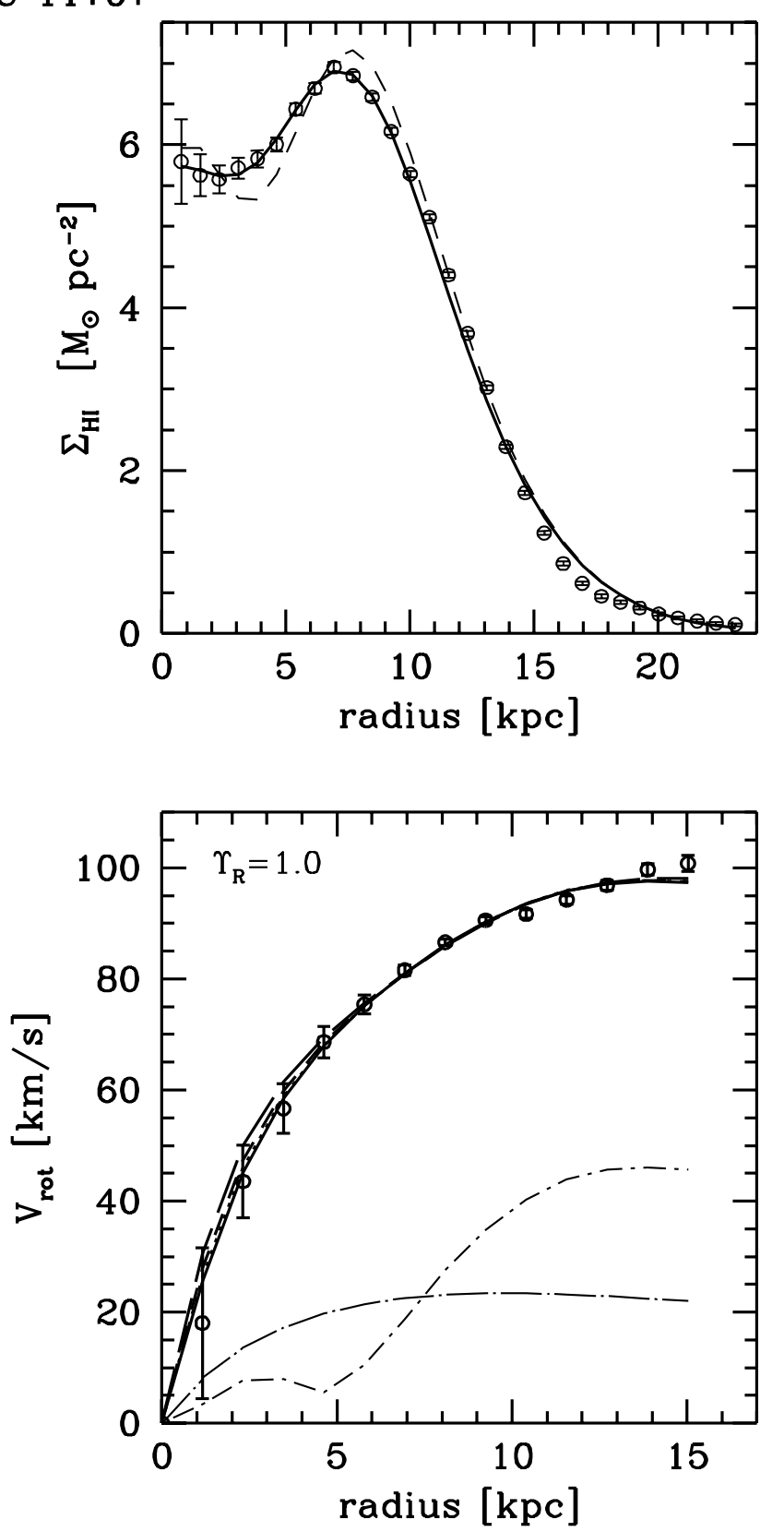

Figure A15. Same as Figure A1 but for UGC 11707 
UGC 11861
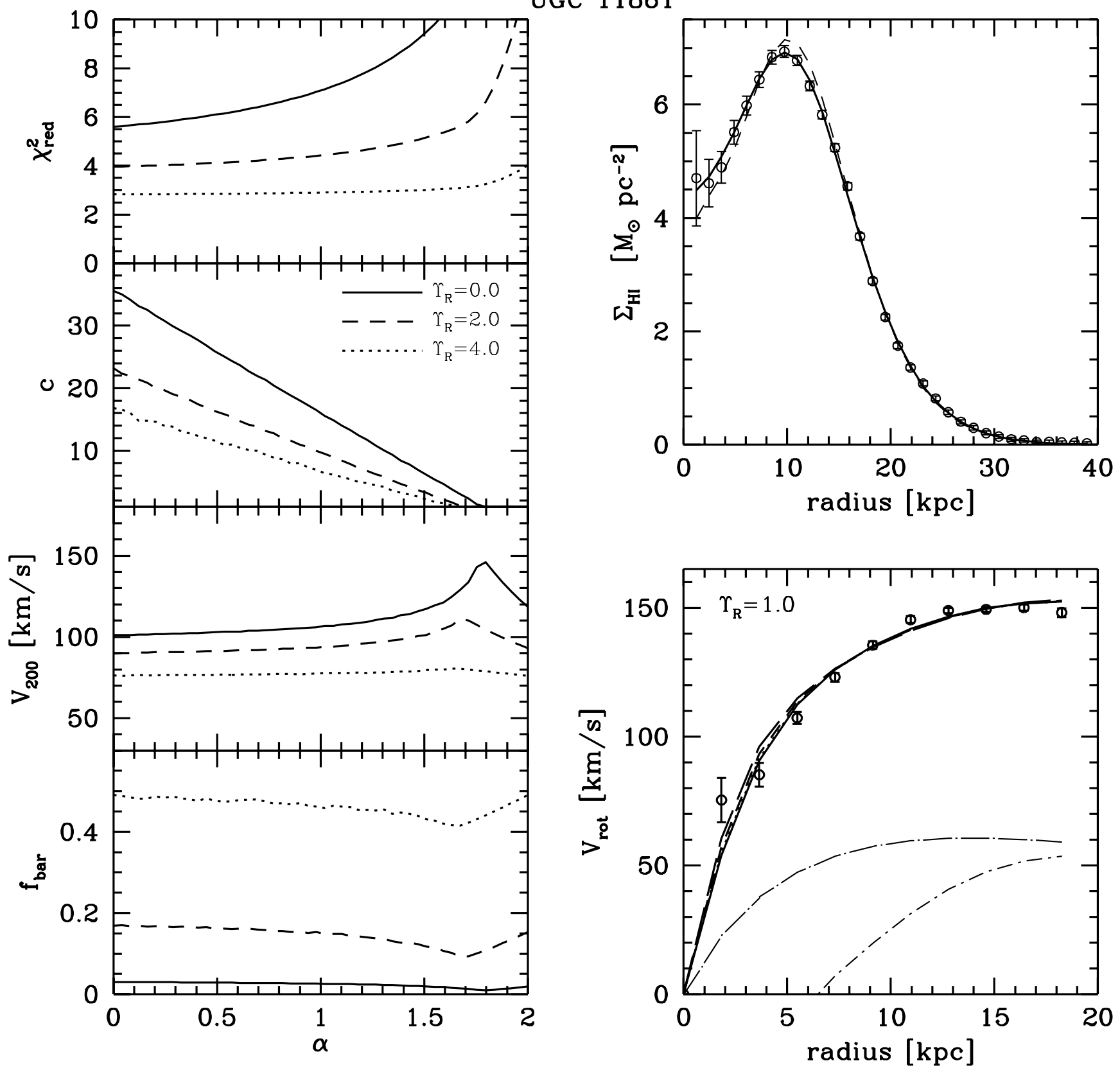

Figure A16. Same as Figure A1 but for UGC 11861 
UGC 12060
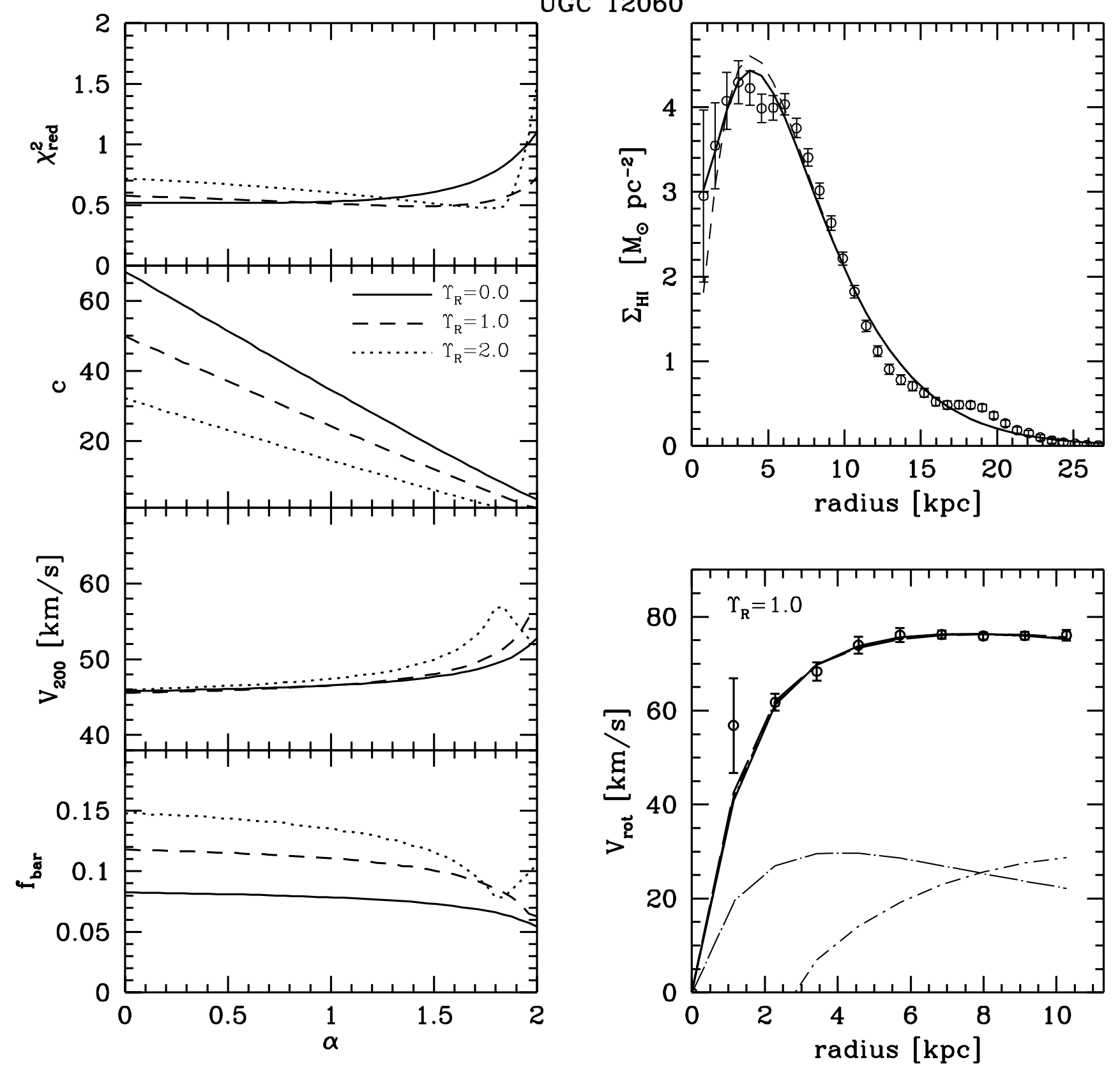

Figure A17. Same as Figure A1 but for UGC 12060 


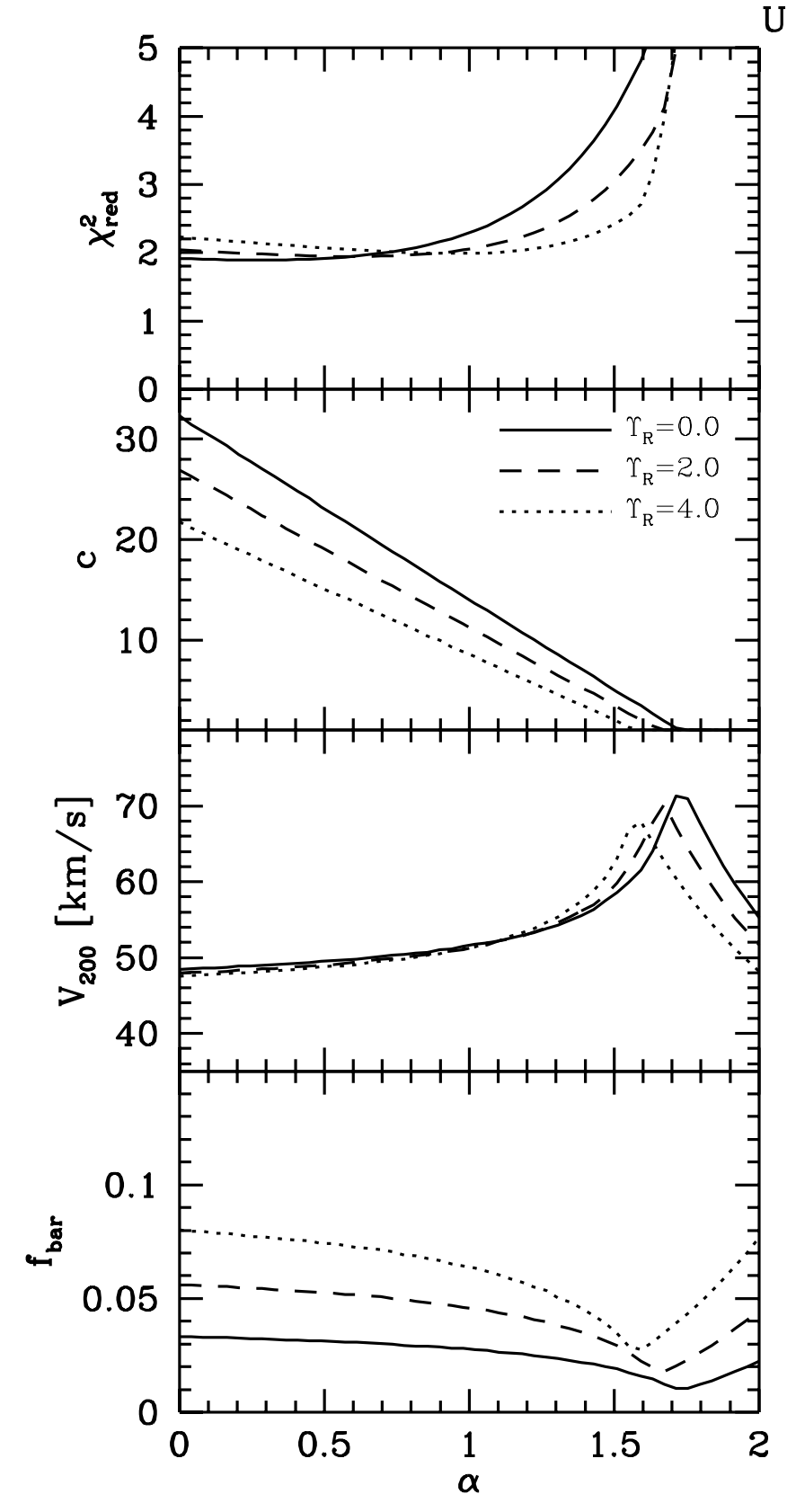

UGC 12632
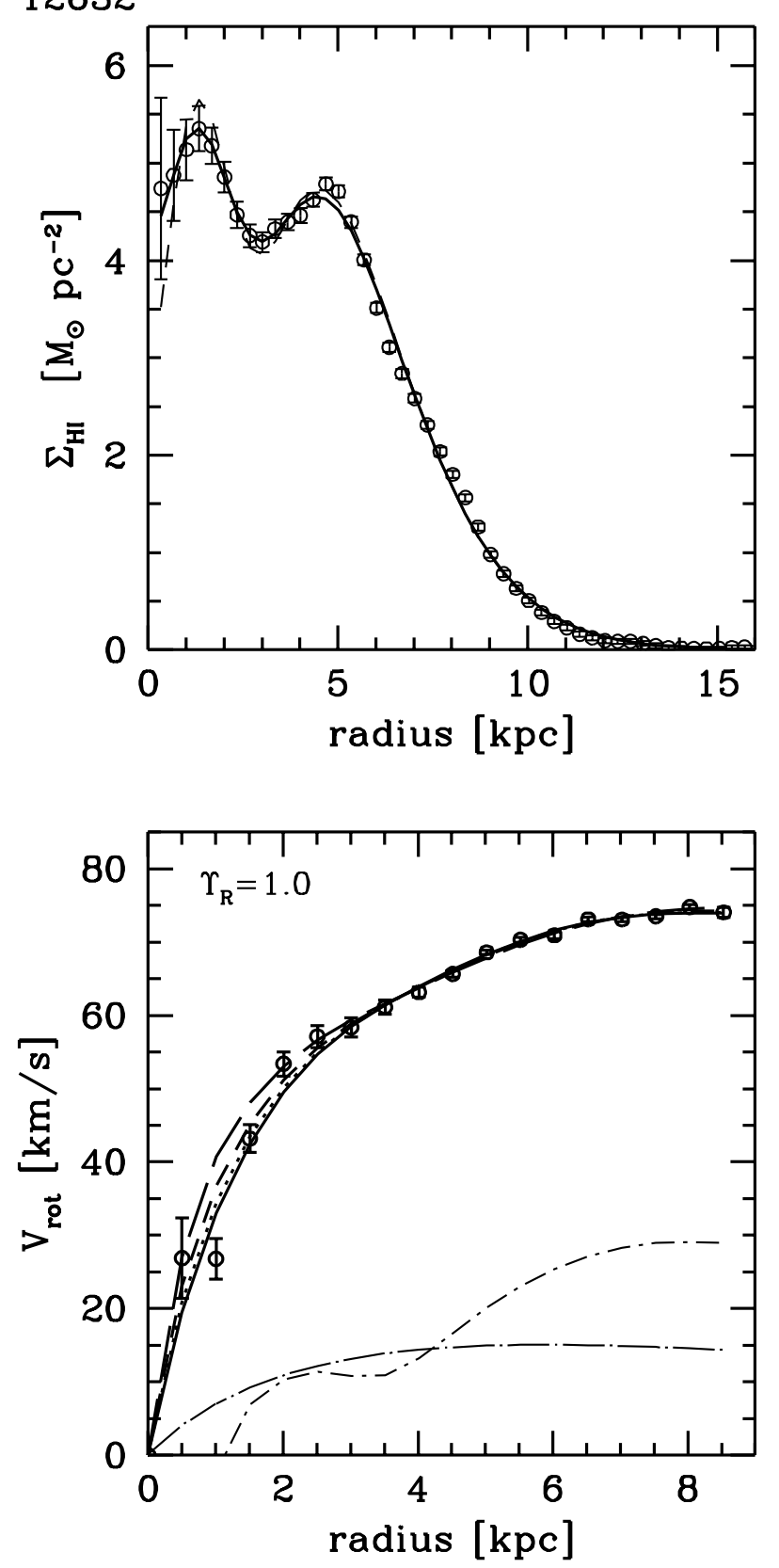

Figure A18. Same as Figure A1 but for UGC 12632 
UGC 12732
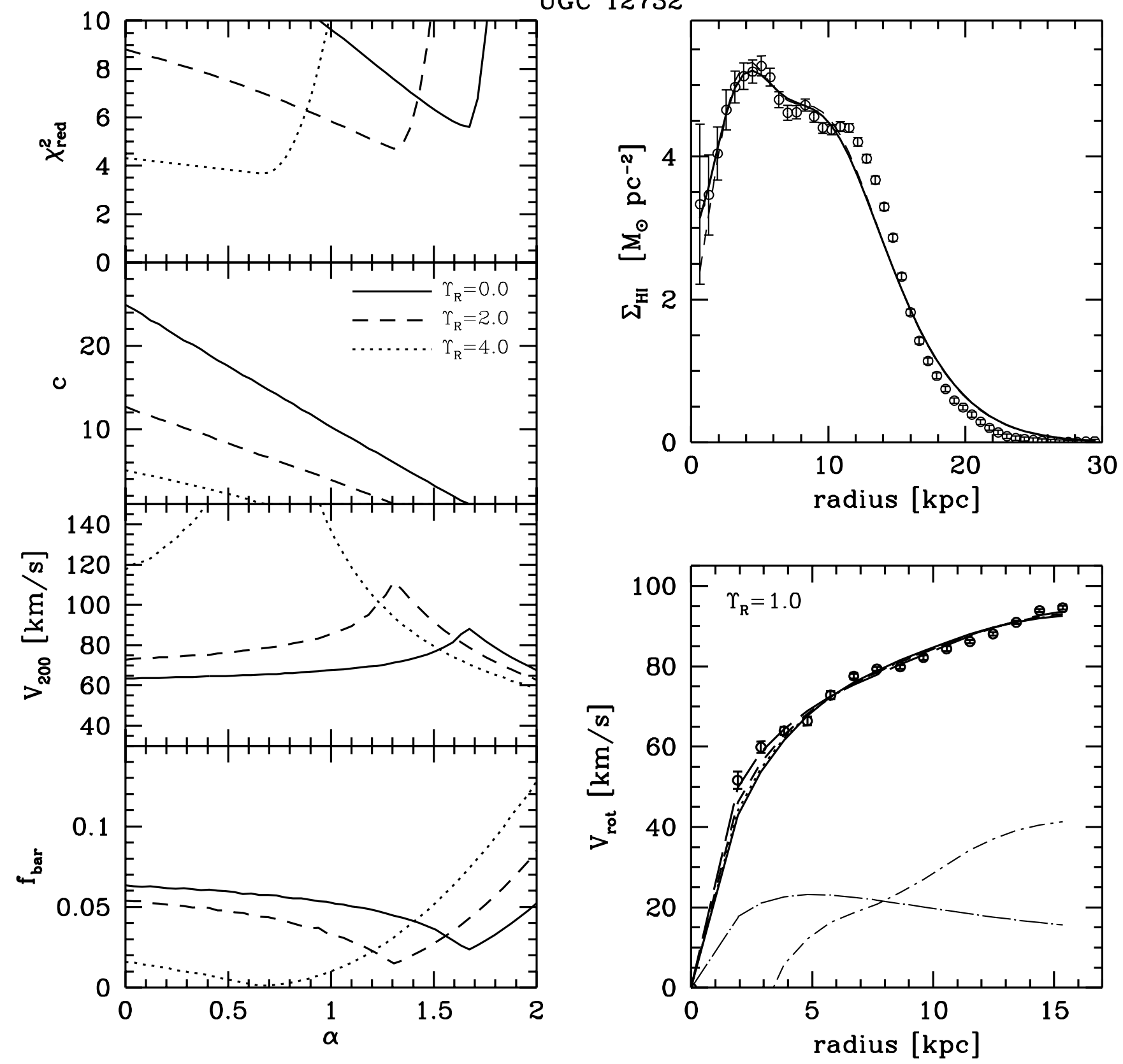

Figure A19. Same as Figure A1 but for UGC 12732 